\title{
Continuous Generalized Gradient Descent
}

\author{
Cun-Hui ZHANG
}

\begin{abstract}
This article derives characterizations and computational algorithms for continuous general gradient descent trajectories in high-dimensional parameter spaces for statistical model selection, prediction, and classification. Examples include proportional gradient shrinkage as an extension of LASSO and LARS, threshold gradient descent with right-continuous variable selectors, threshold ridge regression, and many more with proper combinations of variable selectors and functional forms of a kernel. In all these problems, general gradient descent trajectories are continuous piecewise analytic vector-valued curves as solutions to matrix differential equations. We show the monotonicity and convergence of the proposed algorithms in the loss or negative likelihood functions. We prove that approximations of continuous solutions via infinite series expansions are computationally more efficient and accurate compared with discretization methods. We demonstrate the applicability of our algorithms through numerical experiments with real and simulated datasets.
\end{abstract}

Key Words: Classification; Matrix differential equation; Regression; Regularized optimization.

\section{INTRODUCTION}

An effective regularization strategy in high-dimensional regression and model selection problems is using validation methods to choose a suitable point in a trajectory or a family of trajectories in a parameter space (Hoerl and Kennard 1970; Breiman 1996; Tibshirani 1996; Friedman and Popescu 2004). The trajectory, which could also arise from statistical learning algorithms such as SVM (Vapnik 1996; Wahba, Lin, and Zhang 2000) and boosting (Schapire 1990; Freund and Schapire 1996, 1997; Friedman, Hastie, and Tibshirani 2000; Friedman 2001), typically provides increasing goodness-of-fit to the training data as it traverses from simple sparse models to more complicated ones. In this article, we consider generalized gradient descent (GGD) trajectories as continuous piecewise $\mathbb{R}^{p}$-valued solutions of differential equations.

Cun-Hui Zhang is Professor, Department of Statistics, Hill Center, Busch Campus, Rutgers University, Piscataway, NJ 08816 (E-mail: czhang@stat.rutgers.edu).

\footnotetext{
(c) 2007 American Statistical Association, Institute of Mathematical Statistics, and Interface Foundation of North America
}

Journal of Computational and Graphical Statistics, Volume 16, Number 3, Pages 1-21

DOI: $10.1198 / 106186007 X 238846$ 
Consider a dataset containing response variables $y_{i}$ and covariates $\mathbf{x}_{i} \in \mathbb{R}^{p}$. Let

$$
L(\boldsymbol{\beta})=\sum_{i=1}^{n} w_{i} L_{i}\left(y_{i}, \mathbf{x}_{i}^{\mathrm{tr}} \boldsymbol{\beta}\right), \quad w_{i}>0,
$$

be a weighted loss or negative log-likelihood function, with parameter $\boldsymbol{\beta} \in \mathbb{R}^{p}$ and losses $L_{i}\left(y_{i}, a\right)$ convex in $a$. We provide characterizations and computational algorithms for certain entire GGD trajectories $\boldsymbol{\beta}(t)=\left(\beta_{1}(t), \ldots, \beta_{p}(t)\right)^{t r}$ of the form

$$
d \boldsymbol{\beta}(t)=\mathbf{Q}(t) \mathbf{g}(t) v(d t), \quad t_{0} \leq t \leq T,
$$

with $T \leq \infty$, where $\mathbf{Q}(t)$ is a nonnegative-definite matrix-valued kernel, $v(d t)$ is a measure in $\mathbb{R}$, and $\mathbf{g}(t)=\left(g_{1}(t), \ldots, g_{p}(t)\right)^{t r}$ is the negative gradient of $L(\boldsymbol{\beta})$ at the point $\boldsymbol{\beta}=$ $\boldsymbol{\beta}(t)$,

$$
\mathbf{g}(t)=-(\nabla L)(\boldsymbol{\beta}(t)), \quad \nabla L=\frac{\partial L}{\partial \boldsymbol{\beta}}(\boldsymbol{\beta}) \in \mathbb{R}^{p} .
$$

For continuous $v(d t)$, the nonnegativity of the kernel $\mathbf{Q}(t)$ ensures the monotonicity of the trajectory in the loss $L(\boldsymbol{\beta})$ (Section 5). This monotonicity typically guarantees the convergence of $\boldsymbol{\beta}(t)$ to the minimizer of $L(\cdot)$, but the minimizer typically over fits the data for large $p$. Thus, for the purpose of finding and understanding suitable regularized solutions in the trajectory $\boldsymbol{\beta}(t)$, it is very useful to compute and analyze its entire path.

Friedman and Popescu (2004) proposed the discrete threshold gradient descent (TGD, see Section 2), as a general path-finding tool for model selection and regularization in statistical classification and prediction. They pointed out that the TGD and a number of other path finding algorithms are special cases of the discrete GGD

$$
\boldsymbol{\beta}(t+\Delta ; \Delta)-\boldsymbol{\beta}(t ; \Delta)=\Delta \mathbf{h}(t), \quad t / \Delta=0,1,2, \ldots,
$$

with $\mathbf{h}^{t r}(t) \mathbf{g}(t) \geq 0$. Although (1.4) certainly holds for $\mathbf{h}(t)=\mathbf{Q}(t) \mathbf{g}(t)$ in (1.2) with the discrete $v(d t)=\Delta, t=0, \Delta, 2 \Delta, \ldots$, this article focuses on the characterization and computation of the entire path of continuous piecewise analytic solutions of (1.2) with continuous $v(d t)$.

In Section 2 we study the limit of TGD as $\Delta \rightarrow 0+$ and introduce a nested threshold gradient descent (NTGD) algorithm inspired by the TGD as a simple continuous GGD (1.2). In Section 3, we consider the $\ell_{1}$ penalty and proportional gradient shrinkage (PGS) trajectories as its extension. General methodologies of generating GGD are presented in Section 4, including penalty-driven trajectories, entrance and exit rules for variable selection, and functional forms of the kernel $\mathbf{Q}(t)$ of the GGD (1.2). Threshold ridge regression (TRR) is described in Section 4.4 as another specific example. In Section 5, we prove the monotonicity and consistency of continuous GGD and provide explicit solutions of a general class of GGD. In Section 6, we propose forward recursive algorithms for the computation of the GGD based on the solutions in Sections 4 and 5, and we prove that our algorithms are computationally more efficient and accurate than the recursive algorithms corresponding to equally spaced discretizations of the measure $v(d t)$ with a small step 
size $\Delta$ in (1.4). Section 7 contains some numerical examples to demonstrate the proposed algorithms, and Section 8 provides some discussion.

A number of recent articles have studied the characterization and computation of the entire continuous path of trajectories for statistical prediction and classification. Consider

$$
\boldsymbol{\beta}(t)=\underset{\boldsymbol{\beta}}{\arg \min }\left\{L(\boldsymbol{\beta})+t^{-1} J(\boldsymbol{\beta})\right\},
$$

where $J(\boldsymbol{\beta})$ is a convex penalty function. For the squared loss $L_{i}\left(y_{i}, a\right)=\left(y_{i}-a\right)^{2} / 2$ in (1.1), Osborne, Presnell, and Turlach (2000a, 2000b) and Efron, Hastie, Johnstone, and Tibshirani (2004) proposed algorithms for the exact computation of LASSO (Tibshirani 1996). Rosset and Zhu (2003) extended these results and the more general least angle regression (LARS) of Efron et al. (2004). They pointed out that the trajectory $\boldsymbol{\beta}(t)$ in (1.5) is piecewise linear and can be efficiently computed in the following two cases:

1. $L_{i}\left(y_{i}, a\right)$ are piecewise linear in $a \in \mathbb{R}$ and $J(\boldsymbol{\beta})=\|\boldsymbol{\beta}\|^{2} / 2$ is the $\ell_{2}$-penalty with the Euclidean distance $\|\boldsymbol{\beta}\|$;

2. $L_{i}\left(y_{i}, a\right)$ are piecewise mixed quadratic or linear as in (2.8) (p. 6) and $J(\boldsymbol{\beta})=\|\boldsymbol{\beta}\|_{1}$.

Case 1 includes (kernel) SVM as in Hastie, Rosset, Tibshirani, and Zhu (2004), while Case 2 includes robust LASSO with Huber's loss function; see (4.2) (p. 9). In Proposition 2 in Section 4.1, we prove that (1.5) is a GGD (and thus is monotone in loss by Theorem 1 in Section 5) under the condition that the number of active variables is no greater than the rank of the second derivative of $t L(\boldsymbol{\beta})+J(\boldsymbol{\beta})$ with respect to these variables. Thus, our results provide a unified treatment for the computation of gradient-driven (1.2) and penalty driven (1.5) trajectories.

Throughout this article, $\mathbf{G}^{-1}$ for symmetric matrices $\mathbf{G}$ is defined as the generalized inverse $\sum_{c_{j} \neq 0} c_{j}^{-1} \mathbf{v} \mathbf{v}^{t r}$, where $\sum_{j} c_{j} \mathbf{v} \mathbf{v}^{t r}$ is the eigenvalue decomposition of $\mathbf{G}$.

\section{THE LIMIT OF DISCRETE TGD AND CONTINUOUS NESTED TGD}

Regularized gradient descent is characterized by

$$
d \boldsymbol{\beta}(t)=\mathbf{P}(t) \mathbf{g}(t) v(d t), \quad \mathbf{P}(t)=\mathbf{P}^{2}(t)=\operatorname{diag}(\mathbf{P}(t)),
$$

with a variable selector $\mathbf{P}(t)$ (0-1 diagonal) as the kernel $\mathbf{Q}(t)$ in (1.2), so that the trajectory $\mathbf{P}(t) \boldsymbol{\beta}(t)$ for the selected variables traverses exactly in the direction of the projected negative gradient $\mathbf{P}(t) \mathbf{g}(t)$. This formulation includes both discrete and continuous versions with proper choice of $v(d t)$ and a wide range of possible variable selectors $\mathbf{P}(t)$.

In this section, we show that the limit of the discrete TGD with infinitesimal steps is not necessarily a regularized gradient descent trajectory as defined in (2.1). We then introduce the nested threshold gradient descent (NTGD) as a simple example of (1.2) with continuous $v(d t)$. 


\subsection{The Limit of Threshold Gradient Descent}

The discrete TGD of Friedman and Popescu (2004) computes $\boldsymbol{\beta}(t ; \Delta)$ recursively with

$$
\boldsymbol{\beta}(t+\Delta ; \Delta)=\boldsymbol{\beta}(t ; \Delta)+\Delta \mathbf{P}_{\tau}(t) \mathbf{g}(t), \quad \mathbf{P}_{\tau}(t)=\operatorname{diag}\left(I\left\{\left|g_{j}(t)\right| \geq \tau g^{*}(t)\right\}\right),
$$

at $t=k \Delta, k=0,1,2, \ldots$, where $g^{*}(t)=\max _{j \leq p}\left|g_{j}(t)\right|$ is the maximum gradient. The tuning parameter $\tau$ allows different levels of diversity in variable selection, with the most stringent $\tau=1$ corresponding to a discrete approximation of the LARS and the least stringent $\tau=0$ corresponding to the ordinary gradient descent without regularization.

The discrete TGD is a form of regularized gradient descent (2.1) with variable selector $\mathbf{P}(t)=\mathbf{P}_{\tau}(t)$ and the uniform discrete measure $v(\{\Delta k\})=\Delta$ in (1.2). Does (2.2) converge to a continuous piecewise analytic solution of (2.1) with any variable selector $\mathbf{P}(t)$ ? The answer is "Not in general" as demonstrated by Example 1. In fact, the example shows that the differential equation $d \boldsymbol{\beta}(t)=\mathbf{P}_{\tau}(t) \mathbf{g}(t) d t$ does not have a continuous piecewise analytic solution for certain values of $\mathbf{y}$ in the linear model

$$
L(\boldsymbol{\beta})=\|\mathbf{y}-\mathbf{X} \boldsymbol{\beta}\|^{2} / 2, \quad \mathbf{g}(t)=\mathbf{G}\left(\boldsymbol{\beta}_{\mathrm{LSE}}-\boldsymbol{\beta}(t)\right), \quad \mathbf{G}=\mathbf{X}^{t r} \mathbf{X} .
$$

Example 1. Consider (2.3) with $p=2$ and $\mathbf{G}=\mathbf{X}^{t r} \mathbf{X}=\left(\begin{array}{ll}1 & \rho \\ \rho & 1\end{array}\right)$. Let $\boldsymbol{\beta}(t ; \Delta), t=k \Delta$, be the discrete TGD in (2.2) with $0<\rho<\tau<1$ and small $\Delta>0$. Since $\mathbf{g}(t)=$ $\mathbf{X}^{t r} \mathbf{y}-\mathbf{G} \boldsymbol{\beta}(t ; \Delta),(2.2)$ implies $\mathbf{g}(t+\Delta)-\mathbf{g}(t)=-\mathbf{G P}_{\tau}(t) \mathbf{g}(t) \Delta+O\left(\Delta^{2}\right)$, so that

$$
\frac{g_{1}(t+\Delta)}{g_{2}(t+\Delta)} \approx \frac{g_{1}(t)-\Delta\left\{g_{1}(t)+\rho g_{2}(t)\right\}}{g_{2}(t)-\Delta\left\{\rho g_{1}(t)+g_{2}(t)\right\}} \approx \frac{g_{1}(t)}{g_{2}(t)}-\rho \Delta\left\{1-\left(\frac{g_{1}(t)}{g_{2}(t)}\right)^{2}\right\}
$$

with error $O\left(\Delta^{2}\right)$ for $\min \left\{\left|g_{1}(t)\right|,\left|g_{2}(t)\right|\right\} \geq \tau g^{*}(t)$, while for $\left|g_{1}(t)\right|<\tau\left|g_{2}(t)\right|$

$$
\frac{g_{1}(t)}{g_{2}(t)}=\frac{g_{1}(t)-\Delta \rho g_{2}(t)}{g_{2}(t)-\Delta g_{2}(t)}=\frac{g_{1}(t)}{g_{2}(t)}+\Delta\left\{\frac{g_{1}(t)}{g_{2}(t)}-\rho\right\}+O\left(\Delta^{2}\right) .
$$

Thus, for $\rho<g_{1}(t) / g_{2}(t)<1$, (2.2) always drives $\mathbf{g}(t)$ towards the line $g_{1}(t) / g_{2}(t)=\tau$, since $0<\rho<\tau$. It follows that the limit of discrete TGD becomes a straight line satisfying $g_{1}(t) / g_{2}(t)=\tau$ after it hits the line. However, in view of (2.4) and (2.3), continuous regularized gradient descent must satisfy $\boldsymbol{\beta}(t)=\mathbf{G}^{-1}\left\{\mathbf{X}^{t r} \mathbf{y}-\mathbf{g}(t)\right\}$ and

$$
(d / d t)\left\{g_{1}(t) / g_{2}(t)\right\}=-\rho\left[1-\left\{g_{1}(t) / g_{2}(t)\right\}^{2}\right]
$$

when both components of $\boldsymbol{\beta}(t)$ are active, so that $\mathbf{g}(t)$ and $\boldsymbol{\beta}(t)$ are both nonlinear. Therefore, the limit of discrete TGD cannot be of the form $d \boldsymbol{\beta}(t)=\mathbf{P}(t) \mathbf{g}(t) d t$ for any variable selector $\mathbf{P}(t)$. In fact, after the first crossing of $g_{1}(t) / g_{2}(t)$ over level $\tau$, the discrete TGD is dominated by the variable selector $\mathbf{P}_{\tau}(t)$ as $\mathbf{P}_{\tau}(t)$ starts to oscillate regularly between one- and two-dimensinal projections to force $g_{1}(t) / g_{2}(t) \approx \tau$, so that (2.1) does not have a solution with $\mathbf{P}(t)=\mathbf{P}_{\tau}(t)$.

In Figure 1, we plot 64 trajectories for each of the following four procedures: the LASSO/LARS, the gradient descent with $d \boldsymbol{\beta}(t)=\mathbf{g}(t) d t$ [i.e. with the identity $\mathbf{P}(t)=\mathbf{I}$ in (2.1)], the limit of the discrete TGD (2.2) with $\tau=1 / 2$, and the NTGD in (2.6) below with 

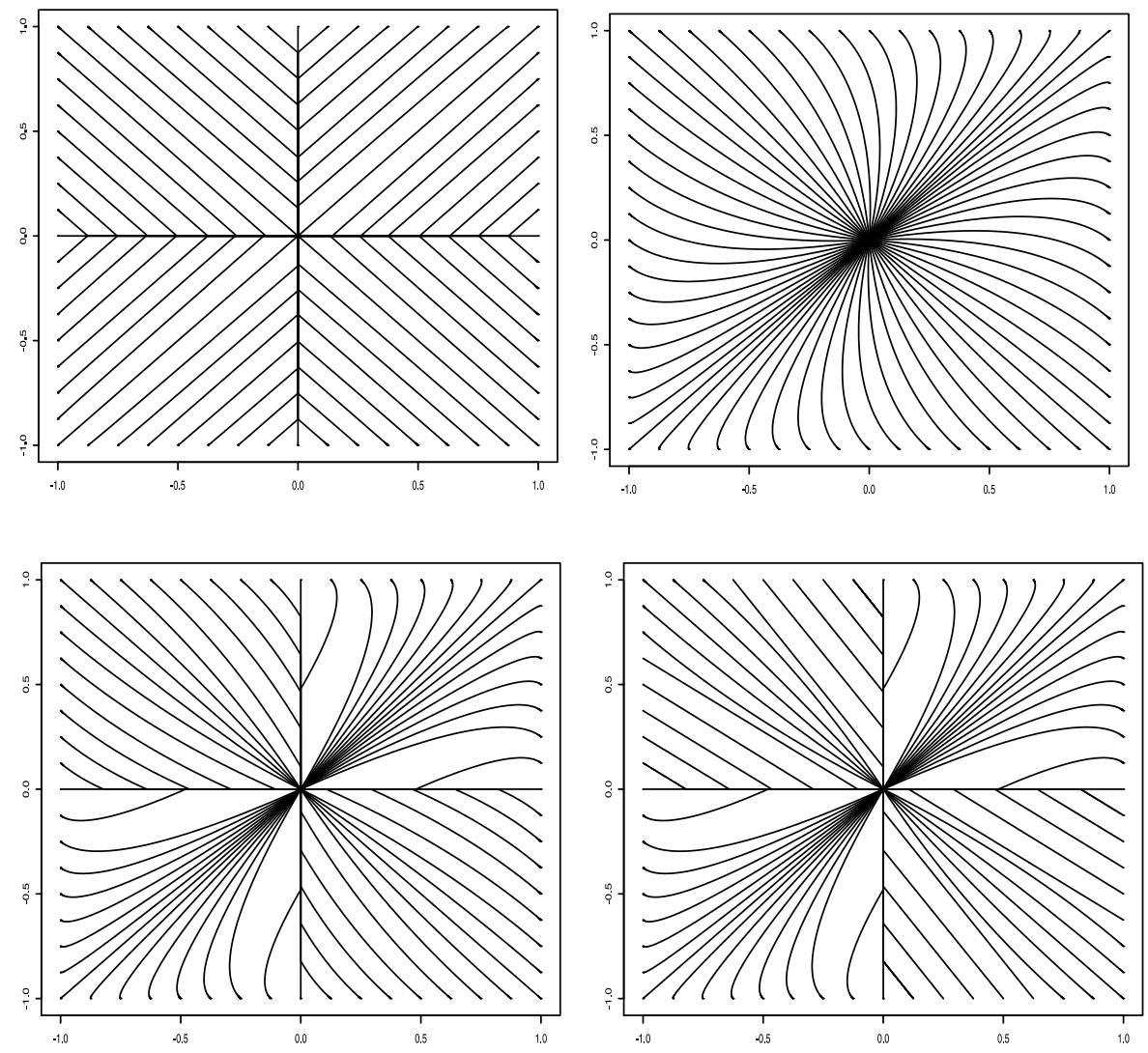

Figure 1. LASSO, GD, limit of TGD, and NTGD, clockwise from top left: Each path is computed with a dataset giving the LSE in the boundary of the square, with the initialization $\boldsymbol{\beta}(0)=0$. LASSO paths are always piecewise linear. GD paths are always nonlinear outside the $45 \%$ lines. Some paths of the limit of TGD are linear outside the axes and the $45 \%$ lines, so that (2.1) fails to hold. The NTGD paths are always nonlinear outside the axes and the $45 \%$ lines.

$\tau=1 / 2$. Each trajectory, beginning from $\boldsymbol{\beta}_{0}$ and ending at the LSE, is based on a realization of $(\mathbf{X}, \mathbf{y})$ in model (2.3) such that $\mathbf{X}^{T} \mathbf{X}=\left(\begin{array}{l}1 \\ \rho \\ \rho\end{array}\right)$ with $\rho=0.3$ and the LSE is in the sets $\{-1,1\} \times\{ \pm k / 8, k=0, \ldots, 8\}$ or $\{ \pm k / 8, k=0, \ldots, 8\} \times\{-1,1\}$ on the boundary of the unit square. When both variables are active, the limit of the discrete TGD yields multiple straight lines in the second and fourth quadrants, while the NTGD always generates nonlinear curves satisfying (2.5) outside the 45-degree trajectories satisfying $\left|\beta_{1}(t)\right|=\left|\beta_{2}(t)\right|$.

For general $p>2$, the discrete TGD still drives $\left|g_{j}(t)\right| / g^{*}(t)$ to $\tau$ for certain $j$ when the negative gradient $\mathbf{g}(t)$ falls into certain regions, but a complete characterization of such patterns and the resulting kernel $\mathbf{Q}(t)$ of (1.2) for its limit is complicated.

\subsection{The Entire Path of NTGD}

We have demonstrated that continuous (2.1) does not always have a solution with the variable selector $\mathbf{P}_{\tau}(t)$ of the discrete TGD (2.2). If we still desire regularized gradient descent with a tuning parameter $\tau$ for different levels of diversity in variable selection, the 
variable selector $\mathbf{P}_{\tau}(t)$ has to be modified to avoid the domination of the variable selector through "instantaneous oscillation" between different sets of active variables as in Example 1. The simplest such modification is a "no-exit" policy with nested sets of active variables in $t$. This leads to the following continuous nested TGD (NTGD):

$$
d \boldsymbol{\beta}(t)=\mathbf{P}_{\tau}^{*}(t) \mathbf{g}(t) d t, \quad \mathbf{P}_{\tau}^{*}(t)=\operatorname{diag}\left(I\left\{\sup _{s \leq t}\left|g_{j}(s)\right| / g^{*}(s) \geq \tau\right\}\right),
$$

with $g^{*}(t)=\max _{j \leq p}\left|g_{j}(t)\right|$. As the kernel $\mathbf{Q}(t)$ in (1.2) and variable selector $\mathbf{P}(t)$ in (2.1), the variable selector $\mathbf{P}_{\tau}^{*}(t)$ clearly forces nested active sets $A(t+\Delta) \subset A(t)$ for all $\Delta>0$, where

$$
A(t)=\left\{j: \inf \left\{\epsilon>0: \beta_{j}(t+\epsilon) \neq \beta_{j}(t)\right\}=0\right\} .
$$

As in the case of the TGD, the NTGD provides $\boldsymbol{\beta}(t)$ with different levels of diversity in variable selection via the tuning parameter $\tau \in[0,1]$. Compared with LASSO which achieves the maximum local reduction in the loss $L(\boldsymbol{\beta})$ for a given step size $\|\mathbf{P}(t) d \boldsymbol{\beta}\|_{1}$ under the $\ell_{1}$ norm, the NTGD seeks the maximum local reduction in $L(\boldsymbol{\beta})$ for a given step size $\|\mathbf{P}(t) d \boldsymbol{\beta}\|$ under the $\ell_{2}$ norm, i.e. the maximum of $-d L(\boldsymbol{\beta}(t)) /\|\mathbf{P}(t) d \boldsymbol{\beta}(t)\|$ given the active set. As a special case of (2.1), the trajectory $\boldsymbol{\beta}(t)$ of the NTGD always moves in the direction of the negative gradient $\mathbf{g}(t)$ within the active set of variables. Under mild conditions, for example, the "one-at-a-time" condition $\operatorname{rank}\left(\mathbf{P}_{\tau}^{*}(t)\right)-\operatorname{rank}\left(\mathbf{P}_{\tau}^{*}(t-)\right) \leq 1$, the discrete version of the NTGD converges to its continuous version since its variable selector does not oscillate unboundedly many times. In Section 4.2, we describe more general selectors $\mathbf{P}(t)$ which also allow variables to drop out of the active set (2.7).

We provide in the rest of the section an explicit solution of (2.6) along with a computationally efficient algorithm. Consider the loss function $L(\boldsymbol{\beta})$ in (1.1) as a sum of convex piecewise mixed quadratic or linear terms $L_{i}\left(y_{i}, \mathbf{x}_{i}^{t r} \boldsymbol{\beta}\right)$ of the form

$$
L_{i}\left(y_{i}, a\right)=\sum_{k=0}^{2} \widetilde{c}_{i k} a^{k}+\sum_{j=1}^{m_{i}} \sum_{k=0}^{2} c_{i j k}\left(a-a_{i j}\right)_{+}^{k},
$$

with knots $\left\{a_{i j}, j \leq m_{i}\right\}$, where $x_{+}=\max (x, 0)$. For $\mathbf{x}_{i}^{t r} \boldsymbol{\beta}\left(t_{0}\right) \neq a_{i j}, j \leq m_{i}, \forall i \leq n$, there exists a neighborhood of $t_{0}$ such that $L(\boldsymbol{\beta}(t))$ is a quadratic function of $\boldsymbol{\beta}(t)$ with coefficients depending only on data and $\boldsymbol{\beta}\left(t_{0}\right)$. This implies

$$
\mathbf{g}(t)=\mathbf{v}_{k}-\mathbf{G}_{k} \boldsymbol{\beta}(t), \quad t_{k}<t<t_{k+1} .
$$

with certain vectors $\mathbf{v}_{k} \in \mathbb{R}^{p}$ and nonnegative-definite $p \times p$ matrices $\mathbf{G}_{k}$, where $t_{k}$ are the discontinuities of $\mathbf{P}_{\tau}^{*}(t)$ or the hitting times with $\mathbf{x}_{i}^{t r} \boldsymbol{\beta}\left(t_{k}\right)=a_{i j}$ for certain $(i, j)$. Set $\mathbf{P}_{k}=\mathbf{P}_{\tau}^{*}\left(t_{k}\right)$ and $\mathbf{H}_{k}=\mathbf{P}_{k} \mathbf{G}_{k} \mathbf{P}_{k}$. We will prove in Theorem 2 in Section 6 that

$$
\boldsymbol{\beta}(t)=\boldsymbol{\beta}\left(t_{k}\right)+\left(t-t_{k}\right) F^{*}\left(-\left(t-t_{k}\right) \mathbf{H}_{k}\right) \mathbf{P}_{k} \mathbf{g}\left(t_{k}\right), t_{k} \leq t \leq t_{k+1},
$$

is the unique continuous solution of (2.6), where $F^{*}(\lambda)=\left(e^{\lambda}-1\right) / \lambda=\sum_{m=1}^{\infty} \lambda^{m-1} / m$ !. Actually, it is not hard to verify using infinite series expansions that (2.10) satisfies (2.6) with

$$
\mathbf{P}_{k} \mathbf{g}(t)=\exp \left(-\left(t-t_{k}\right) \mathbf{H}_{k}\right) \mathbf{P}_{k} \mathbf{g}\left(t_{k}\right), \quad t \in\left[t_{k}, t_{k+1}\right)
$$


Formally, (2.10) can be computed using the following algorithm with any initialization $\boldsymbol{\beta}\left(t_{0}\right)$ at $t_{0}$.

NTGD Algorithm: Set $F^{*}(\lambda)=\left(e^{\lambda}-1\right) / \lambda$; while $t_{k}<T$

$$
\begin{aligned}
\boldsymbol{\beta}(t) & \leftarrow \boldsymbol{\beta}\left(t_{k}\right)+\left(t-t_{k}\right) F^{*}\left(-\left(t-t_{k}\right) \mathbf{H}_{k}\right) \mathbf{P}_{k} \mathbf{g}\left(t_{k}\right), t_{k}<t \leq T \\
t_{k+1} & \leftarrow \min _{i, j} \min \left\{t>t_{k}: \mathbf{x}_{i}^{t r} \boldsymbol{\beta}(t)=a_{i j}\right\} \\
t_{k+1} & \leftarrow \min \left[t_{k+1}, \min _{j} \min \left\{t>t_{k}:\left|g_{j}(t)\right|=\tau g^{*}(t), \beta_{j}(s)=\beta_{j}\left(t_{0}\right) \forall s<t\right\}\right]
\end{aligned}
$$

Here are two possibilities of completing (2.12). The first is

$$
F^{*}\left(-\left(t-t_{k}\right) \mathbf{H}_{k}\right) \mathbf{P}_{k} \mathbf{g}\left(t_{k}\right)=\sum_{j} \mathbf{u}_{j k} F^{*}\left(-\left(t-t_{k}\right) \lambda_{j k}\right) \mathbf{u}_{j k}^{t r} \mathbf{P}_{k} \mathbf{g}\left(t_{k}\right)
$$

via the singular value decomposition (SVD) $\mathbf{H}_{k}=\mathbf{P}_{k} \mathbf{G}_{k} \mathbf{P}_{k}=\sum_{j} \lambda_{j k} \mathbf{u}_{j k} \mathbf{u}_{j k}^{t r}$, while the second is the approximation

$$
\boldsymbol{\beta}(t) \leftarrow \boldsymbol{\beta}\left(t_{k}\right)+\left(t-t_{k}\right) \sum_{m=1}^{N_{k}}\left(-\left(t-t_{k}\right) \mathbf{H}_{k}\right)^{m-1} \mathbf{P}_{k} \mathbf{g}\left(t_{k}\right) / m !
$$

through the infinite series expansion of $F^{*}(\lambda)$, see (6.8)-(6.11) and Theorem 3 in Section 6.

\section{THE $\ell_{1}$ PENALTY AND PROPORTIONAL GRADIENT SHRINKAGE}

In this section, we consider (1.5) with the $\ell_{1}$ penalty $J(\boldsymbol{\beta})=\|\boldsymbol{\beta}\|_{1}=\sum_{j=1}^{p}\left|\beta_{j}\right|$ and a class of closely related GGD (1.2) with certain proportional gradient shrinkage (PGS) property. We show that the trajectory

$$
\boldsymbol{\beta}(t)=\arg \min \left\{\boldsymbol{\beta} \in \mathbb{R}^{p}: L(\boldsymbol{\beta})+t^{-1}\|\boldsymbol{\beta}\|_{1}\right\}
$$

is a solution of (1.2) characterized by this PGS property and a special variable selector. The PGS trajectory allows more general variable selectors and they contain as a special case the linear portion of the limit of the TGD in Example 1 of Section 2.1.

Let $L(\boldsymbol{\beta})$ be the loss or negative log-likelihood function in (1.1) and define

$$
\mathbf{G}(t)=\left.\frac{\partial}{\partial \boldsymbol{\beta}}\left(\frac{\partial}{\partial \boldsymbol{\beta}}\right)^{t r} L(\boldsymbol{\beta})\right|_{\boldsymbol{\beta}=\boldsymbol{\beta}(t)} .
$$

Since both $L(\boldsymbol{\beta})$ and $\|\boldsymbol{\beta}\|_{1}$ are convex in $\boldsymbol{\beta}$, it follows from the Kuhn-Tucker criterion that $\boldsymbol{\beta}(t)$ is a solution of (3.1) iff $\boldsymbol{\beta}(t)=\mathbf{0}$ for $t \leq t_{0}$ and

$$
\mathbf{P}(t) \mathbf{g}(t)=\mathbf{S}(t) t^{-1} \mathbf{1}_{p}, \quad g^{*}(t)=\max _{j}\left|g_{j}(t)\right|=t^{-1}, \quad t \geq t_{0},
$$

where $\mathbf{P}(t)=\operatorname{diag}\left(I\left\{\beta_{j}(t) \neq 0\right\}\right)$ is the variable selector, $\mathbf{g}(t)$ is the negative gradient as in (1.3), $\mathbf{S}(t)=\operatorname{diag}\left(\operatorname{sgn}\left(\beta_{j}(t)\right)\right)$ is the sign matrix of $\boldsymbol{\beta}(t)$ with the convention $\operatorname{sgn}(0)=0$, 
$t_{0}=1 /\|\nabla L(\mathbf{0})\|_{\infty}$, and $\mathbf{1}_{p}=(1, \ldots, 1)^{t r} \in \mathbb{R}^{p}$. Let $t_{0}<t_{1}<\cdots<t_{k}<\cdots$ be the discontinuities of $\mathbf{P}(t), \mathbf{S}(t)$ or $d \mathbf{g}(t) / d t$. Since $\mathbf{P}(t)$ and $\mathbf{S}(t)$ are constant diagonal matrices in $t_{k}<t<t_{k+1}$, we find by (3.2) and (3.3)

$$
\mathbf{P}(t) \mathbf{G}(t) d \boldsymbol{\beta}(t)=-\mathbf{P}(t) d \mathbf{g}(t)=\mathbf{S}(t) t^{-2} \mathbf{1}_{p} d t=\mathbf{P}(t) \mathbf{g}(t) t^{-1} d t .
$$

Since $d \boldsymbol{\beta}(t)=\mathbf{P}(t) d \boldsymbol{\beta}(t)$, it follows that

$$
d \boldsymbol{\beta}(t)=\{\mathbf{P}(t) \mathbf{G}(t) \mathbf{P}(t)\}^{-1} \mathbf{g}(t) v(d t), \quad t_{k}<t<t_{k+1},
$$

with $v(t)=t^{-1} d t$. This is GGD (1.2) with the kernel $\mathbf{Q}(t)=\{\mathbf{P}(t) \mathbf{G}(t) \mathbf{P}(t)\}^{-1}$. The following proposition gives the PGS interpretation of (3.4) and its relationship to (3.1).

Proposition 1. Let $\boldsymbol{\beta}(t)$ be a piecewise analytic trajectory satisfying (1.2) and $\mathbf{P}(t)$ be variable selectors satisfying $\mathbf{P}(t) d \boldsymbol{\beta}(t)=d \boldsymbol{\beta}(t)$. Suppose $\boldsymbol{\beta}(t)$ is analytic and $\mathbf{P}(t)$ is continuous in intervals $\left[t_{k}, t_{k+1}\right)$. Then, (3.4) holds iff the following PGS property holds

$$
\mathbf{P}(t) \mathbf{g}(t)=\exp \left\{-v\left(\left[t_{k}, t\right)\right)\right\} \mathbf{P}\left(t_{k}\right) \mathbf{g}\left(t_{k}\right), \quad t_{k} \leq t<t_{k+1} .
$$

Moreover, for $v(d t)=t^{-1} d t$ and $t_{0}=1 /\|\nabla L(\mathbf{0})\|_{\infty}=\inf \{t:\|\boldsymbol{\beta}(t)\| \neq 0\}$, (3.5) for all $k \geq 0$ is equivalent to (3.1) for $t \geq t_{0}$ iff for all $t_{k}<t<t_{k+1}$ and $k \geq 0$

$$
\mathbf{P}(t)=\operatorname{diag}\left(\max \left[I\left\{\left|g_{j}(t)\right|=g^{*}(t), \operatorname{sgn}\left(g_{j}(t)\right)=\operatorname{sgn}\left(\beta_{j}(t)\right)\right\}, I\left\{\beta_{j}\left(t_{k}\right) \neq 0\right\}\right]\right),
$$

provided that $\beta_{j}(t)$ does not change sign in $\left(t_{k}, t_{k+1}\right)$.

Proof: Since $\mathbf{P}(t) d \boldsymbol{\beta}(t)=d \boldsymbol{\beta}(t)$ and $\mathbf{P}(t) \mathbf{G}(t) d \boldsymbol{\beta}(t)=-\mathbf{P}(t) d \mathbf{g}(t)$, (3.4) is equivalent to $\mathbf{P}(t) d \mathbf{g}(t)=-\mathbf{P}(t) \mathbf{g}(t) v(d t)$, which has (3.5) as the unique solution. Thus, (3.4) and (3.5) are equivalent. Now, consider $v(d t)=t^{-1} d t$ and the given boundary condition at $t_{0}$. Since (3.1) implies (3.4), it suffices to show by induction that (3.5) and (3.6) give (3.1). The initial value $\boldsymbol{\beta}\left(t_{0}\right)=\mathbf{0}$ is assumed with $g^{*}\left(t_{0}\right)=t_{0}^{-1}$. Now, assume (3.3) holds at $t=t_{k}$. For the specific $\mathbf{P}(t)$, (3.5) implies

$$
\mathbf{P}(t) \mathbf{g}(t)=\left(t_{k} / t\right) \mathbf{P}(t) \mathbf{g}\left(t_{k}\right)=\left(t_{k} / t\right) g^{*}\left(t_{k}\right) \mathbf{P}(t) \mathbf{S}(t) \mathbf{1}_{p}=t^{-1} \mathbf{P}(t) \mathbf{S}(t) \mathbf{1}_{p},
$$

and $g^{*}(t) / t=g^{*}\left(t_{k}\right) / t_{k}=1$ for $t_{k}<t<t_{k+1}$. It remains to show $\mathbf{P}(t) \mathbf{S}(t)=\mathbf{S}(t)$ for $t_{k}<t<t_{k+1}$. If $\beta_{j}\left(t_{k}\right) \neq 0$, then $\mathbf{P}(t)$ selects the $j$ th variable by definition. If $\beta_{j}\left(t_{k}\right)=0$ and $\mathbf{P}(t)$ does not select the $j$ th variable, then $d \beta_{j}(t)=0$ and $\beta_{j}(t)=\beta_{j}\left(t_{k}\right)=0$. Thus, $\mathbf{P}(t)$ selects all variables $j$ with $\beta_{j}(t) \neq 0$, that is, $\mathbf{P}(t) \mathbf{S}(t)=\mathbf{S}(t)$.

Proposition 1 provides a PGS interpretation of the LASSO and LARS through the analysis of the matrix differential equation (3.4), with extensions to more general loss functions $L(\boldsymbol{\beta})$ and variable selectors $\mathbf{P}(t)$. The solution of (3.4) for $\boldsymbol{\beta}(t)$ is nontrivial for general loss $L(\boldsymbol{\beta})$ even though $\mathbf{g}(t)$ is explicitly given in (3.5). If (2.9) holds as in model (2.8), with $\mathbf{G}(t)=\mathbf{G}\left(t_{k}\right)=\mathbf{G}_{k}$ and $\mathbf{P}(t)=\mathbf{P}\left(t_{k}\right)=\mathbf{P}_{k}$ in $\left(t_{k}, t_{k+1}\right)$, (3.4) and (3.5) give the solution

$$
\boldsymbol{\beta}(t)=\boldsymbol{\beta}\left(t_{k}\right)+\int_{t_{k}}^{t} \exp \left\{-v\left(\left[t_{k}, x\right)\right)\right\} d x\left\{\mathbf{P}_{k} \mathbf{G}_{k} \mathbf{P}_{k}\right\}^{-1} \mathbf{g}\left(t_{k}\right)
$$


for $t_{k} \leq t \leq t_{k+1}$ via the continuity of $\boldsymbol{\beta}(t)$. The value of $t_{k+1}$ here is typically determined from the analytic form of the $k$ th piece of $\boldsymbol{\beta}(t)$, while the solution of the $k$ th piece of $\boldsymbol{\beta}(t)$ in (3.7) is based on the initialization $\left\{t_{k}, \boldsymbol{\beta}\left(t_{k}\right), \mathbf{g}\left(t_{k}\right), \mathbf{P}_{k}, \mathbf{G}_{k}\right\}$ for the piece. We note that this solution of the special GGD (3.4) is valid for almost all right-continuous (i.e., piecewise constant) variables selectors $\mathbf{P}(t)$. For example, if $\mathbf{P}_{\tau}(t)$ of (2.2) is used with $0<\tau<1$, then the active set is nested (i.e., $\mathbf{P}(t) \uparrow$ in $t$ ) and $\left|g_{j}(t)\right| / g^{*}(t)$ is either $\tau$ or 1 for active $j$ and less than $\tau$ otherwise. In the linear model (2.3) with constant $\mathbf{G}(t)=\mathbf{G}$, the LARS (Efron et al. 2004) trajectory is the solution of (3.4) with nested variable selector $\mathbf{P}_{1}=\operatorname{diag}\left(I\left\{\left|g_{j}(t)\right|=g^{*}(t)\right\}\right)$ [i.e., $\tau=1$ in (2.2)]. However, the equi-angular property of LARS no longer holds for $\mathbf{P}_{\tau}$ with $\tau<1$. We note that since the LARS is a GGD, it is monotone in the loss $L(\boldsymbol{\beta})$ (see Section 5).

\section{MORE EXAMPLES OF GGD}

We first show in Proposition 2 that penalty driven trajectories (1.5) can be written as (1.2) under local smoothness conditions on the loss and penalty functions. We then discuss certain families of variable selectors and kernels in (1.2). In Section 4.4, we give explicit description of the TRR as another example of GGD.

\subsection{Penalty-Driven Trajectories}

We show in this section that (1.2) contains a rich class of penalty-driven trajectories (1.5) with different combinations of the loss and penalty functions. For example, the (kernel) SVM (Vapnik 1996; Wahba, Lin, and Zhang 2000) uses

$$
L_{i}\left(y_{i}, a\right)=\left(1-y_{i} a\right)_{+}, \quad y_{i} \in\{-1,1\}
$$

in (1.1), as $L(\boldsymbol{\beta})=0$ with (4.1) implies the separation of $\left\{\mathbf{x}_{i}: y_{i}=1\right\}$ and $\left\{\mathbf{x}_{i}: y_{i}=-1\right\}$ by the hyperplane $\left\{\mathbf{x}: \mathbf{x}^{t r} \boldsymbol{\beta}=1\right\}$. For Huber's robust estimation and prediction,

$$
L_{i}\left(y_{i}, a\right)=\max \left\{\left(y_{i}-a\right)^{2} / 2, \gamma_{i}\left|y_{i}-a\right|-\gamma_{i}^{2} / 2\right\}
$$

with trim levels $\gamma_{i}$. Commonly used penalty functions include the $\ell_{1}$-penalty $J(\boldsymbol{\beta})=$ $\sum_{j=1}^{p}\left|\beta_{j}\right|$ and general quadratic penalty $J(\boldsymbol{\beta})=\boldsymbol{\beta}^{\mathrm{tr}} \mathbf{K} \boldsymbol{\beta} / 2$ with a nonnegative-definite matrix K (e.g., ridge regression and kernel SVM). Friedman et al. (2000) interpreted AdaBoost as a gradient-based incremental search in an additive model.

Let $A(t)$ be the active set at time $t$ as in (2.7). We assume that both the loss function $L(\boldsymbol{\beta})$ and the penalty function $J(\boldsymbol{\beta})$ are differentiable in $\left\{\beta_{j}: j \in A(t)\right\}$ at $\boldsymbol{\beta}=\boldsymbol{\beta}(t)$ in the sense that

$$
g_{j}(t)=-\left.\frac{\partial}{\partial \beta_{j}} L(\boldsymbol{\beta})\right|_{\boldsymbol{\beta}=\boldsymbol{\beta}(t)}, \quad \kappa_{j}(t)=\left.\frac{\partial}{\partial \beta_{j}} J(\boldsymbol{\beta})\right|_{\boldsymbol{\beta}=\boldsymbol{\beta}(t)}, \quad j \in A(t),
$$

are all defined when $A(t)=A(t-)$. The optimality at $\boldsymbol{\beta}=\boldsymbol{\beta}(t)$ in (1.5) then leads to

$$
g_{j}(t)=t^{-1} \kappa_{j}(t), \quad j \in A(t) .
$$


Rosset and Zhu (2003) pointed out the following fact: $\boldsymbol{\beta}(t)$ can be explicitly solved through (4.4) when $\left\{g_{j}(t), \kappa_{j}(t), t \in A\left(t_{k}\right)\right\}$ are all linear in $\boldsymbol{\beta}(t)$ as functions in individual intervals $t_{k}<t<t_{k+1}$ with $A(t)=A\left(t_{k}\right)$, see (2.9).

Identity (4.4) can be further explored to establish the connection between (1.5) and (1.2). Suppose $g_{j}(t)$ and $\kappa_{j}(t)$ are piecewise differentiable such that

$$
\mathbf{P}(t) d \mathbf{g}(t)=-\mathbf{P}(t) \mathbf{G}(t) \mathbf{P}(t) d \boldsymbol{\beta}(t), \quad \mathbf{P}(t) d \boldsymbol{\kappa}(t)=\mathbf{P}(t) \mathbf{K}(t) \mathbf{P}(t) d \boldsymbol{\beta}(t),
$$

where $\mathbf{P}(t)=\operatorname{diag}(I\{j \in A(t)\})$ and $\boldsymbol{\kappa}(t)=\left(\kappa_{1}(t), \ldots, \kappa_{p}(t)\right)^{t r}$, with the convention $\kappa_{j}(t)=0$ for $j \notin A(t)$ if necessary. Typically, components of $\mathbf{G}(t)$ and $\mathbf{K}(t)$ are respectively the second partial derivatives of $L(\boldsymbol{\beta})$ and $J(\boldsymbol{\beta})$ at $\boldsymbol{\beta}=\boldsymbol{\beta}(t)$.

Proposition 2. Let $\mathbf{P}(t)=\operatorname{diag}(I\{j \in A(t)\})$ be the projection to active components of $\boldsymbol{\beta}$, with the active set $A(t)$ in (2.7). Suppose the piecewise differentiability conditions (4.3) and (4.5) hold in $\left(t_{k}, t_{k+1}\right)$. Then,

$$
\mathbf{P}(t)\{t \mathbf{G}(t)+\mathbf{K}(t)\} \mathbf{P}(t) d \boldsymbol{\beta}(t)=\mathbf{P}(t) \mathbf{g}(t) d t, t_{k}<t<t_{k+1} .
$$

If in addition $\mathbf{P}(t)\{t \mathbf{G}(t)+\mathbf{K}(t)\} \mathbf{P}(t)$ is of the same rank as $\mathbf{P}(t)$, then (1.2) holds with

$$
d \boldsymbol{\beta}(t)=[\mathbf{P}(t)\{t \mathbf{G}(t)+\mathbf{K}(t)\} \mathbf{P}(t)]^{-1} \mathbf{g}(t) d t, t_{k}<t<t_{k+1} .
$$

Proposition 2 follows immediately from the differentiation of $\mathbf{P}(t)\{\boldsymbol{\kappa}(t)-t \mathbf{g}(t)\}=0$, the vector form of (4.4), at the points $t$ with $\mathbf{P}(t-)=\mathbf{P}(t)$. Condition (4.7) is necessary for (1.5) but it is not sufficient and thus more general. In particular, it allows the combination of (4.7) with any right-continuous variable selectors. See Sections 4.2 and 4.4.

Remark. If $J(\boldsymbol{\beta})$ is piecewise linear (e.g., the $\ell_{1}$ penalty), then $\mathbf{P}(t) \mathbf{K}(t)=0$ and (4.7) becomes (3.4) for PGS. If $L(\boldsymbol{\beta})$ is piecewise linear and $J(\boldsymbol{\beta})=\boldsymbol{\beta}^{t r} \mathbf{K} \boldsymbol{\beta} / 2$, then $\mathbf{P}(t) \mathbf{G}(t)=0$ and (4.7) yields (1.2) with $\mathbf{Q}(t)=\{\mathbf{P}(t) \mathbf{K P}(t)\}^{-1}$ and $v(d t)=d t$ as in kernel SVM.

\subsection{Variable Selectors}

Although the variable selector of a GGD trajectory can be written as

$$
\mathbf{P}(t)=\operatorname{diag}(I\{j \in A(t)\})
$$

with the active set $A(t)$ in (2.7), it is often defined directly as a function of the trajectory $\boldsymbol{\beta}(t)$ and the gradient $\mathbf{g}(t)$. Since $d \boldsymbol{\beta}(t)=\mathbf{P}(t) d \boldsymbol{\beta}(t)$, we typically consider kernels satisfying $\mathbf{P}(t) \mathbf{Q}(t)=\mathbf{Q}(t) \mathbf{P}(t)$, so that

$$
d \boldsymbol{\beta}(t)=\mathbf{P}(t) \mathbf{Q}(t) \mathbf{P}(t) \mathbf{g}(t) v(d t)
$$

holds as an alternative form of (1.2). 
We have shown in Example 1 of Section 2.1 that a solution of (1.2) may not exist when the variable selector $\mathbf{P}(t)$ dominates the kernel $\mathbf{Q}(t)$. To avoid this situation, we require that the variable selector be right-continuous. One such variable selector is the nested variable selector $P_{\tau}^{*}(t)$ of (2.6). Here we describe a family of "easy-in-hard-out" (EIHO) rightcontinuous variable selectors

$$
\mathbf{P}(t)=\operatorname{diag}\left(\max \left[I\{j \notin A(t-)\} \delta_{j, \tau}^{(\text {in })}(t), I\{j \in A(t-)\}\left\{1-\delta_{j, \tilde{\tau}, \epsilon}^{(\text {out })}(t)\right\}\right]\right),
$$

with the entrance policy $\delta_{j, \tau}^{(\mathrm{in})}(t)=I\left\{\left|g_{j}(t)\right| \geq \tau g^{*}(t)\right\}$ as in (2.2) and the exit policy

$$
\delta_{j, \widetilde{\tau}, \epsilon}^{\text {(out) }}(t)=I\left\{\left|g_{j}(t)\right| \leq \widetilde{\tau} g^{*}(t) \text { and }\left|\boldsymbol{\beta}_{j}(t)\right| \leq \epsilon\right\}, \quad \tilde{\tau} \leq \tau \leq 1,
$$

where $A(t)$ is the active set in (2.7).

The variable selector (4.9) allows the $j$ th variable to enter the active set when $\left|g_{j}(t)\right|$ $/ g^{*}(t)$ is "large" and to exit the active set when $\left|g_{j}(t)\right| / g^{*}(t)$ and $\left|\beta_{j}(t)\right|$ are both "small", with a larger threshold $\tau$ for the entrance policy. In the simplest case of $\tilde{\tau}=\epsilon<0$, $\delta_{j, \widetilde{\tau}, \epsilon}^{(\text {out }}=0$ for all $t$, so that (4.9) becomes the nested $P_{\tau}^{*}(t)$ in (2.6). Another interesting case of (4.9) is $1 \geq \tau>\widetilde{\tau} \approx 1$ and $0<\epsilon \approx 0$, which approximates the variable selector (3.6) for the $\ell_{1}$ penalty.

Since $\mathbf{g}(t)$ and $\boldsymbol{\beta}(t)$ are continuous in $t$, for $\tilde{\tau}<\tau$ (4.9) does not allow a variable to exit the active set immediately after it enters the active set, and this holds uniformly in compact intervals of $t$. Thus, (4.9) is right-continuous. A sensible choice of $\epsilon$ is a certain fraction of the median of the absolute values of the optimal $\beta_{j}$ in univariate models, for example, a fraction of $\operatorname{med}\left(\left|\mathbf{x}_{j}^{t r} \mathbf{y}\right| /\left\|\mathbf{x}_{j}\right\|^{2}\right)$ in the linear model (2.3) with $\mathbf{x}_{j}$ being columns of $\mathbf{X}$.

A variation of (4.9) is the "hard-in-hard-out" (HIHO) variable selector which allows the $j$ th variable to enter the active set only when the $j$ th absolute gradient is greater than the maximum gradient for the active variables by a factor. That is to use

$$
\delta_{j, \tau}^{(\mathrm{in})}(t)=I\left\{\left|g_{j}(t)\right| \geq \tau g_{*}(t)\right\}, \quad \delta_{j, \widetilde{\tau}, \epsilon}^{\text {(out })}(t)=I\left\{\left|g_{j}(t)\right| \leq \tilde{\tau} g_{*}(t),\left|\boldsymbol{\beta}_{j}(t)\right| \leq \epsilon\right\},
$$

in (4.9) with $\tilde{\tau} \leq 1<\tau$ for $A(t-) \neq \emptyset$, where $g_{*}(t)=\max _{j \in A(t-)}\left|g_{j}(t)\right|$. As in the case of EIHO, the HIHO variable selector is also right continuous. A motivation of HIHO is to reduce the bias of $\boldsymbol{\beta}(t)$ given the selected model.

\subsection{Functional Gradient Descent Kernels}

Assume the variable selector $\mathbf{P}(t)$ is right-continuous. As in the derivation of (4.5), under certain differentiability conditions on $L(\boldsymbol{\beta}),(1.2)$ implies

$$
d \mathbf{g}(t)=\mathbf{G}(t) d \boldsymbol{\beta}(t)=\mathbf{G}(t) \mathbf{P}(t) \mathbf{Q}(t) \mathbf{g}(t) d t, \quad t_{k}<t<t_{k+1} .
$$

For $\mathbf{Q}(t)=\mathbf{P}(t)$, we have used (4.11) to find the solution (2.10) for the NTGD with the nested variable selector. For the $\ell_{1}$ penalty, $\mathbf{Q}(t)=\{\mathbf{P}(t) \mathbf{G}(t) \mathbf{P}(t)\}^{-1}$ as in (3.4). Thus, it makes sense to consider functional gradient descent kernels of the form

$$
\mathbf{Q}(t)=q(\mathbf{P}(t) \mathbf{G}(t) \mathbf{P}(t))
$$


for general variable selectors $\mathbf{P}(t)$ and nonnegative functions $q(\lambda)$ with $q(0)=0$. For example, we may consider mixture kernels of the form

$$
q(\lambda)=w \lambda^{\alpha_{1}}+(1-w) \lambda^{\alpha_{2}}, \quad 0 \leq w \leq 1, \alpha_{j} \in \mathbb{R},
$$

with the convention $\lambda^{\alpha}=0$ for $\lambda=0>\alpha$. This includes $q(\lambda)=1$ for the TGD/NTGD and $q(\lambda)=\lambda^{-1}$ for the LASSO/LARS/PGS. We note that if $\operatorname{rank}(\mathbf{P}(t))=\operatorname{rank}(\mathbf{P}(t) \mathbf{G}(t)$ $\mathbf{P}(t)),(4.12)$ provides a piecewise linear trajectory $\boldsymbol{\beta}(t)$ iff $q(\lambda)=\lambda^{-1}$.

\subsection{Threshold Ridge Regression}

Threshold ridge regression (TRR) is the combination of the ridge regression path with threshold variable selectors, for example, the $\mathbf{P}_{\tau}^{*}$ in (2.6) for nested TRR, or the more general EIHO or HIHO selectors in (4.9) and (4.10). Suppose the loss function $L(\boldsymbol{\beta})$ satisfies (2.8) so that the gradient $\mathbf{g}(t)$ is piecewise linear in $\boldsymbol{\beta}(t)$ as in (2.9). Then, the differential equation of TRR can be written in the form (1.2) as

$$
d \boldsymbol{\beta}(t)=\mathbf{Q}(t) \mathbf{g}(t) v(d t), \quad \mathbf{Q}(t)=\left\{\mathbf{P}_{k}\left(t \mathbf{G}_{k}+\mathbf{K}_{k}\right) \mathbf{P}_{k}\right\}^{-1}, \quad t_{k} \leq t<t_{k+1},
$$

with nonnegative-definite matrices $\mathbf{K}_{k}$. Here we may choose a richer set $\left\{t_{k}\right\}$ in (4.14) than the set $\left\{t_{k}\right\}$ in (2.9) to allow $\mathbf{K}_{k}$ to change at continuities of $\mathbf{P}(t)$. For $v(d t)=d t$, (4.14) corresponds to the penalty $t^{-1} \boldsymbol{\beta}^{t r} \mathbf{P}_{k} \mathbf{K}_{k} \mathbf{P}_{k} \boldsymbol{\beta} / 2$ in the $k$-th piece in view of Proposition 2, but discrete or other continuous $v(d t)$ are also allowed here.

For $v(d t)=d t$, the well known explicit solution of the ridge regression path yields the solution of (4.14) with the formula for $t \in\left[t_{k}, t_{k+1}\right]$

$$
\boldsymbol{\beta}(t)=\boldsymbol{\beta}\left(t_{k}\right)-\left\{\mathbf{Q}(t) \mathbf{Q}^{-1}\left(t_{k}\right)-\mathbf{P}_{k}\right\}\left(\mathbf{P}_{k} \mathbf{G}_{k} \mathbf{P}_{k}\right)^{-1} \mathbf{P}_{k} \mathbf{g}\left(t_{k}\right) .
$$

This can be directly derived as follows. By (2.9) and (4.14), $(d / d t) \mathbf{P}_{k} \mathbf{g}(t)=-\mathbf{G}_{k}^{*} \mathbf{Q}(t) \mathbf{g}(t)$ with $\mathbf{G}_{k}^{*}=\mathbf{P}_{k} \mathbf{G}_{k} \mathbf{P}_{k}$. Since $\mathbf{Q}^{-1}\left(t_{k}\right) \mathbf{Q}(t)=\mathbf{G}_{k}^{*} \mathbf{Q}(t) \mathbf{Q}^{-1}\left(t_{k}\right)\left(\mathbf{G}_{k}^{*}\right)^{-1}$ and $(d / d t) \mathbf{Q}(t)=$ $-\mathbf{Q}(t) \mathbf{G}_{k}^{*} \mathbf{Q}(t), \mathbf{P}_{k} \mathbf{g}(t)=\mathbf{Q}^{-1}\left(t_{k}\right) \mathbf{Q}(t) \mathbf{P}_{k} \mathbf{g}\left(t_{k}\right)$ is the explicit solution of $\mathbf{P}_{k} \mathbf{g}(t)$. This and (4.14) lead to the solution (4.15) due to

$$
(d / d t) \boldsymbol{\beta}(t)=\mathbf{Q}(t) \mathbf{Q}^{-1}\left(t_{k}\right) \mathbf{Q}(t) \mathbf{P}_{k} \mathbf{g}\left(t_{k}\right)=-(d / d t)\left(\mathbf{G}_{k}^{*}\right)^{-1} \mathbf{Q}^{-1}\left(t_{k}\right) \mathbf{Q}(t) \mathbf{P}_{k} \mathbf{g}\left(t_{k}\right)
$$

and $\left(\mathbf{G}_{k}^{*}\right)^{-1} \mathbf{Q}^{-1}\left(t_{k}\right) \mathbf{Q}(t)=\mathbf{Q}(t) \mathbf{Q}^{-1}\left(t_{k}\right)\left(\mathbf{G}_{k}^{*}\right)^{-1}$.

\section{MONOTONICITY, CONSISTENCY, AND EXPLICIT SOLUTION OF CONTINUOUS GGD}

We consider in this section analytic properties of solutions of (1.2). We prove the monotonicity of $L(\boldsymbol{\beta}(t))$ in $t$ for the GGD (1.2) and under a mild condition the convergence of $L(\boldsymbol{\beta}(t))$ to the minimum loss. We then derive an explicit solution of (1.2) under a set of sufficient conditions. We assume throughout the sequel that $v\left(\left[t_{0}, t\right]\right)$ is a continuous function of $t$. 
Theorem 1. Let $L_{*}=\min _{\mathbf{b}} L(\mathbf{b})$ and $\left\{\boldsymbol{\beta}(t), t_{0} \leq t<T\right\}$ be a solution of (1.2) with a nonnegative definite kernel $\mathbf{Q}(t)$. Suppose the gradient $\mathbf{g}(t)$ in (1.3) is piecewise continuous in $t$. Then, the loss $L(\boldsymbol{\beta}(t))$ is decreasing in $t$ along the trajectory $\boldsymbol{\beta}(t)$. Moreover, if $\mathbf{g}^{t r}(t) \mathbf{Q}(t) \mathbf{g}(t) \geq c_{*}(t)\|\mathbf{g}(t)\|_{\infty}^{\gamma}$ for certain $\gamma>0$ and function $c_{*}(t)$ with $\int_{t_{0}}^{T} c_{*}(t) v(d t)=$ $\infty$, then $L(\boldsymbol{\beta}(t)) \rightarrow L_{*}$ as $t \rightarrow T$-, provided the boundedness of the set $\left\{\mathbf{b}: L(\mathbf{b})=L_{*}\right\}$.

Remark. In Efron et al. (2004), the monotonicity is referred to as that of the path components $\beta_{j}(t)$ as functions of $t$. For GGD, this monotonicity condition is equivalent to requiring the agreement of $d \beta_{j}(t)$ and $\beta_{j}(T)$ in sign as part of the variable selector $\mathbf{P}(t)$.

Proof: Since $v(d t)$ is continuous, the nonnegativity of the kernel $\mathbf{Q}(t)$ implies

$$
\frac{d L(\boldsymbol{\beta}(t))}{d v(t)}=-\mathbf{g}^{t r}(t) \frac{d \boldsymbol{\beta}(t)}{d v(t)}=-\mathbf{g}^{t r}(t) \mathbf{Q}(t) \mathbf{g}(t) \leq 0
$$

in view of (1.3). Thus, $L(\boldsymbol{\beta}(t))$ is decreasing in $t$. To prove $L(\boldsymbol{\beta}(t)) \rightarrow \min _{\beta} L(\boldsymbol{\beta})$, we assume $L(\boldsymbol{\beta}(T-))>-\infty$. If the lower bound with $c_{*}(t)$ holds, we have

$$
L\left(\boldsymbol{\beta}\left(t_{0}\right)\right)-L(\boldsymbol{\beta}(t))=\int_{t_{0}}^{t}\{\mathbf{g}(t)\}^{t r} \mathbf{Q}(t) \mathbf{g}(t) v(d t) \geq \int_{t_{0}}^{t} c_{*}(x)\left\{g^{*}(x)\right\}^{\gamma} v(d x) .
$$

Since $\int_{t_{0}}^{T} c_{*}(t) v(d t)=\infty$, we have liminf $g^{*}(t)=0$ as $t \rightarrow T-$. This gives the convergence of $L(\boldsymbol{\beta}(t))$ to the minimum due to the convexity of $L(\boldsymbol{\beta})$.

We provide explicit solutions of the GGD Equation (1.2) under the following set of conditions.

Condition A: Given an initialization $\boldsymbol{\beta}\left(t_{0}\right)$ at $t=t_{0}$, there exist points $t_{0}<t_{k}<t_{k+1}$, variable selectors $\mathbf{P}_{k}$ and $p \times p$ matrices $\mathbf{G}_{k}(t)$ and $\mathbf{Q}_{k}(t)$ satisfying the following conditions: (i) $\boldsymbol{\beta}(t)-\boldsymbol{\beta}\left(t_{k}\right)=\mathbf{P}_{k}\left\{\boldsymbol{\beta}(t)-\boldsymbol{\beta}\left(t_{k}\right)\right\}$ for $t_{k} \leq t \leq t_{k+1}$; (ii) $\mathbf{P}_{k} d \mathbf{g}(t)=\mathbf{P}_{k} \mathbf{G}_{k}(t) d \boldsymbol{\beta}(t)$ for $t \in\left(t_{k}, t_{k+1}\right)$; (iii) $\left\{\mathbf{P}_{k} \mathbf{G}_{k}(t) \mathbf{Q}_{k}(t), t_{k} \leq t<t_{k+1}\right\}$ is a family of commutative matrices; (iv) $\mathbf{P}_{k} \mathbf{G}_{k}(t) \mathbf{Q}_{k}(t)$ is right-continuous in $t \in\left[t_{k}, t_{k+1}\right)$; (v) $\mathbf{Q}(t)=\mathbf{Q}_{k}(t)=\mathbf{Q}_{k}(t) \mathbf{P}_{k}$ for $t_{k} \leq t<t_{k+1}$; and (vi) $\left\{\mathbf{P}_{k}, \mathbf{Q}_{k}(t), \mathbf{G}_{k}(t), t \geq t_{k}\right\}$ are determined by $\left\{\boldsymbol{\beta}(t): t \leq t_{k}\right\}$.

Condition A(i) essentially asserts that the active set does not change in $\left[t_{k}, t_{k+1}\right)$, so that $\mathbf{P}(t)=\mathbf{P}_{k}$ in the interval. Condition A(ii) is a local differentiability condition on $L(\boldsymbol{\beta})$ at $\boldsymbol{\beta}=\boldsymbol{\beta}(t)$ and is a consequence of (2.9), see (4.5). Condition $\mathrm{A}(\mathrm{iii})$ is a consequence of (2.9) and (4.12), but it also allows TRR (4.14). Since $\mathbf{P}(t) d \boldsymbol{\beta}(t)=d \boldsymbol{\beta}(t)$, (1.2) can be written as $d \boldsymbol{\beta}=\mathbf{P}(t) \mathbf{Q}(t) \mathbf{g}(t) v(d t)$ as in (4.8). Thus, Condition $\mathrm{A}(\mathrm{v})$ asserts that the increment $d \boldsymbol{\beta}(t)$ depend on the gradient $\mathbf{g}(t)$ only through its components $g_{j}(t)$ with $j$ in the active set. A discrete version of Condition A (vi) for $\mathbf{P}(t)$ and $\mathbf{Q}(t)$ is necessary for the discrete algorithm

$$
\boldsymbol{\beta}\left(x_{j+1} ; \Delta\right) \leftarrow \boldsymbol{\beta}\left(x_{j} ; \Delta\right)+\mathbf{P}\left(x_{j}\right) \mathbf{Q}\left(x_{j}\right) \mathbf{g}\left(x_{j}\right) \Delta .
$$

Since $\mathbf{Q}(t)$ is determined by $\mathbf{G}(t)$ in most examples given earlier, Condition $\mathrm{A}(\mathrm{vi})$ is natural for (1.2) as the limit of (5.1). 
Theorem 2. Suppose Condition A holds with an initialization $\boldsymbol{\beta}\left(t_{0}\right)$ at $t_{0}$. Let $T=$ $\sup _{k} t_{k}$. Then, (1.2) has a unique continuous solution satisfying

$$
\begin{aligned}
\boldsymbol{\beta}(t) & =\boldsymbol{\beta}\left(t_{k}\right)+\int_{t_{k}}^{t} \mathbf{Q}_{k}(s) \mathbf{g}(s) v(d s), \quad t_{k} \leq t \leq t_{k+1}, \\
\mathbf{P}_{k} \mathbf{g}(t) & =\exp \left[\mathbf{P}_{k} \int_{t_{k}}^{t} \mathbf{G}_{k}(s) \mathbf{Q}_{k}(s) v(d s)\right] \mathbf{P}_{k} \mathbf{g}\left(t_{k}\right), \quad t_{k} \leq t<t_{k+1} .
\end{aligned}
$$

Furthermore, if (2.9) holds and $\mathbf{Q}(t)=\mathbf{Q}_{k}$ for $t_{k} \leq t<t_{k+1}$, then

$$
\boldsymbol{\beta}(t)=\boldsymbol{\beta}\left(t_{k}\right)+v\left(\left(t_{k}, t\right]\right) F^{*}\left(-v\left(\left(t_{k}, t\right]\right) \mathbf{H}_{k}\right) \mathbf{Q}_{k} \mathbf{g}\left(t_{k}\right), t_{k}<t \leq t_{k+1},
$$

where $\mathbf{H}_{k}=\mathbf{Q}_{k} \mathbf{G}_{k} \mathbf{P}_{k}$ and $F^{*}(\lambda)=\left(e^{\lambda}-1\right) / \lambda=\sum_{m=1}^{\infty}(m !)^{-1} \lambda^{m-1}$ as in (2.10).

Proof: We shall find the solution by induction. Suppose (5.2) and (5.3) provide the solution in $\left[t_{0}, t_{k}\right]$. Let $\widetilde{\mathbf{H}}_{k}(t)=\mathbf{P}_{k} \mathbf{G}_{k}(t) \mathbf{Q}_{k}(t)$. By Condition A(i), (ii), and (v),

$$
d \mathbf{P}_{k} \mathbf{g}(t)=\mathbf{P}_{k} d \mathbf{g}(t)=\mathbf{P}_{k} \mathbf{G}_{k}(t) d \boldsymbol{\beta}(t)=\widetilde{\mathbf{H}}_{k}(t) \mathbf{P}_{k} \mathbf{g}(t) v(d t)
$$

in $\left[t_{k}, t_{k+1}\right)$ as the right-differentiation, so that (5.3) must hold by Condition A(iii), (iv), and (vi). This also implies (5.2).

Now suppose (2.9) holds and $\mathbf{Q}(t)=\mathbf{Q}_{k}$. Since $\mathbf{P}_{k} \mathbf{Q}_{k} \widetilde{\mathbf{H}}(t)=\mathbf{H}_{k} \mathbf{P}_{k} \mathbf{Q}_{k}$,

$$
\begin{aligned}
\boldsymbol{\beta}(t)-\boldsymbol{\beta}\left(t_{k}\right) & =\mathbf{Q}_{k} \int_{t_{k}}^{t} \exp \left[-v\left(\left(t_{k}, s\right]\right) \widetilde{\mathbf{H}}\left(t_{k}\right)\right] \mathbf{g}\left(t_{k}\right) v(d s) \\
& =\mathbf{Q}_{k} \sum_{m=0}^{\infty} \frac{\left(-\widetilde{\mathbf{H}}\left(t_{k}\right)\right)^{m}}{m !} \int_{t_{k}}^{t} v^{m}\left(\left(t_{k}, s\right]\right) v(d s) \mathbf{g}\left(t_{k}\right) \\
& =\sum_{m=1}^{\infty}(m !)^{-1}\left(-\mathbf{H}_{k}\right)^{m-1} v^{m}\left(\left(t_{k}, t\right]\right) \mathbf{Q}_{k} \mathbf{g}\left(t_{k}\right), t_{k} \leq t<t_{k+1},
\end{aligned}
$$

by (5.2) and (5.3). This is (5.4).

\section{COMPUTATION OF THE ENTIRE GGD TRAJECTORY}

The GGD solution of (1.2) in Theorem 2 can be computed via the Jordan decomposition of $\mathbf{P}_{k} \mathbf{G}_{k}(t) \mathbf{Q}_{k}(t)$ or the infinite series expansion of $F^{*}(\cdot)$, since Condition A(iii) guarantees common basis of the Jordan decompositions of $\mathbf{P}_{k} \mathbf{G}_{k}(t) \mathbf{Q}_{k}(t), t_{k} \leq t<t_{k+1}$. We focus here on the computation of the more explicit (5.4) and (4.15) via singular value decompositions (SVD) or the infinite series. Between the two choices, the GGD algorithm based on SVD is exact and computationally more efficient when the number of active variables is not too large. Moreover, we prove that the GGD algorithm based on infinite series expansions provides computationally more efficient and accurate approximation than discretization of (1.2). 


\subsection{ForWARD GGD ALgORITHMS}

We consider (5.4) in this subsection. Suppose (2.9) holds and $\mathbf{Q}(t)=\mathbf{Q}_{k}$ for $t_{k} \leq t<$ $t_{k+1}$. Let $\mathbf{H}_{k}^{*}=\mathbf{Q}_{k}^{1 / 2} \mathbf{G}_{k} \mathbf{Q}_{k}^{1 / 2}$. Since $\mathbf{Q}_{k}^{1 / 2} \mathbf{H}_{k}^{*}=\mathbf{H}_{k} \mathbf{Q}_{k}^{1 / 2}, F\left(\mathbf{H}_{k}\right) \mathbf{Q}_{k}=\mathbf{Q}_{k}^{1 / 2} F\left(\mathbf{H}_{k}^{*}\right) \mathbf{Q}_{k}^{1 / 2}$ for all functions $F$. Therefore, (5.4) can be computed via the SVD of $\mathbf{Q}_{k}$ and $\mathbf{H}_{k}^{*}$ as follows.

Forward GGD Algorithm I: Set $F^{*}(\lambda)=\left(e^{\lambda}-1\right) / \lambda$; while $t_{k}<T$

$$
\begin{aligned}
& \text { Compute the } \operatorname{SVD} \mathbf{Q}_{k}=\mathbf{U}_{k}^{t r} \operatorname{diag}\left(\lambda_{k j}^{\prime}\right) \mathbf{U}_{k} \\
& \mathbf{H}_{k}^{*} \leftarrow \mathbf{U}_{k}^{t r} \operatorname{diag}\left(\left(\lambda_{k j}^{\prime}\right)^{1 / 2}\right) \mathbf{U}_{k} \mathbf{G}_{k} \mathbf{U}_{k}^{t r} \operatorname{diag}\left(\left(\lambda_{k j}^{\prime}\right)^{1 / 2}\right) \mathbf{U}_{k} \\
& \text { Compute the } \operatorname{SVD} \mathbf{H}_{k}^{*}=\mathbf{V}_{k}^{t r} \operatorname{diag}\left(\lambda_{k j}^{\prime \prime}\right) \mathbf{V}_{k} \\
& \mathbf{f}_{k}(t) \leftarrow \mathbf{V}_{k}^{t r} \operatorname{diag}\left(F^{*}\left(-v\left(\left(t_{k}, t\right]\right) \lambda_{k j}^{\prime \prime}\right)\right) \mathbf{V}_{k} \mathbf{U}_{k}^{t r} \operatorname{diag}\left(\left(\lambda_{k j}^{\prime}\right)^{1 / 2}\right) \mathbf{U}_{k} \mathbf{g}\left(t_{k}\right) \\
& \boldsymbol{\beta}(t) \leftarrow \boldsymbol{\beta}\left(t_{k}\right)+v\left(\left(t_{k}, t\right]\right) \mathbf{U}_{k}^{t r} \operatorname{diag}\left(\left(\lambda_{k j}^{\prime}\right)^{1 / 2}\right) \mathbf{U}_{k} \mathbf{f}_{k}(t), t \geq t_{k} \\
& \text { Compute } t_{k+1} \text { and } \mathbf{P}_{k+1} \text { from } \boldsymbol{\beta}(t), t>t_{k}
\end{aligned}
$$

Algorithm I can be carried out as long as the rank of $\mathbf{P}_{k}$ does not exceed the dimensionality limit for computing the SVD of nonnegative-definite matrices in (6.1) and (6.3). If $\mathbf{P}_{k} \mathbf{G}_{k} \mathbf{P}_{k}$ and $\mathbf{Q}_{k}$ commute, then they and $\mathbf{H}_{k}=\mathbf{H}_{k}^{*}$ share the same eigenvectors with $\mathbf{U}_{k}=\mathbf{V}_{k}$, so that (6.2)-(6.5) can be simplified as

$$
\boldsymbol{\beta}(t) \leftarrow \boldsymbol{\beta}\left(t_{k}\right)+\mathbf{U}_{k}^{t r} \operatorname{diag}\left(F^{*}\left(-v\left(\left(t_{k}, t\right]\right) \lambda_{k j}^{\prime \prime}\right)\right) v\left(\left(t_{k}, t\right]\right) \lambda_{k j}^{\prime} \mathbf{U}_{k} \mathbf{g}\left(t_{k}\right), t \geq t_{k},
$$

where $\operatorname{diag}\left(\lambda_{k j}^{\prime \prime}\right)=\mathbf{U}_{k} \mathbf{H}_{k} \mathbf{U}_{k}^{t r}$. In particular, if (4.12) holds, then $\lambda_{k j}^{\prime}=q\left(\lambda_{k j}\right)$ and $\lambda_{k j}^{\prime \prime}=$ $\lambda_{k j} q\left(\lambda_{k j}\right)$, where $\mathbf{U}^{t r} \operatorname{diag}\left(\lambda_{k j}\right) \mathbf{U}_{k}$ is the SVD of $\mathbf{P}_{k} \mathbf{G}_{k} \mathbf{P}_{k}$ as in (2.15). Alternatively, without computing SVD, (5.4) can be approximated by

$$
\widetilde{\boldsymbol{\beta}}(t ; N)=\boldsymbol{\beta}\left(t_{k}\right)+F_{N}^{*}\left(-v\left(\left(t_{k}, t\right]\right) \mathbf{H}_{k}\right) v\left(\left(t_{k}, t\right]\right) \mathbf{Q}_{k} \mathbf{g}\left(t_{k}\right)
$$

with the partial sum $F_{N}^{*}(\lambda)=\sum_{m=1}^{N} \lambda^{m-1} / m$ !, as in the following algorithm. The computational cost in this approach is of the same order as that of the discrete (5.1) when $N_{k} \Delta \asymp v\left(\left[t_{k}, t_{k+1}\right)\right)$.

Forward GGD Algorithm II: While $t_{k}<T$

$$
\begin{aligned}
& \mathbf{h}_{0} \leftarrow \mathbf{Q}_{k} \mathbf{g}\left(t_{k}\right), \quad \mathbf{f}_{1}^{*} \leftarrow \mathbf{h}_{0} \\
& \text { For }\left(m=1, \ldots, N_{k}-1\right), \\
& \quad\left\{\mathbf{h}_{m} \leftarrow \mathbf{H}_{k} \mathbf{h}_{m-1}, \quad \mathbf{f}_{m+1}^{*} \leftarrow \mathbf{f}_{m}^{*}+\left\{-v\left(\left(t_{k}, t\right]\right)\right\}^{m} \mathbf{h}_{m} /(m+1) !\right\} \\
& \boldsymbol{\beta}(t) \leftarrow \boldsymbol{\beta}\left(t_{k}\right)+v\left(\left(t_{k}, t\right]\right) \mathbf{f}_{N_{k}}^{*}, \quad t \geq t_{k} \\
& \text { Compute } t_{k+1} \text { and } \mathbf{P}_{k+1} \text { from } \boldsymbol{\beta}(t), t>t_{k}
\end{aligned}
$$

\subsection{A ForWARd TRR Algorithm}

The computation of (4.15) is similar to that of (5.4), although $\mathbf{Q}(t)=\mathbf{Q}\left(t_{k}\right)$ fails to hold for TRR. It follows from (4.14) that $\mathbf{Q}(t) \mathbf{Q}^{-1}\left(t_{k}\right)=\left(\mathbf{G}_{k}^{*}\right)^{-1 / 2}\left(t+\mathbf{K}_{k}^{*}\right)^{-1}\left(t_{k}+\mathbf{K}_{k}^{*}\right)\left(\mathbf{G}_{k}^{*}\right)^{1 / 2}$ 
with $\mathbf{G}_{k}^{*}=\mathbf{P}_{k} \mathbf{G}_{k} \mathbf{P}_{k}$ and $\mathbf{K}_{k}^{*}=\left(\mathbf{G}_{k}^{*}\right)^{-1 / 2} \mathbf{K}_{k}\left(\mathbf{G}_{k}^{*}\right)^{-1 / 2}$. Thus, (4.15) can be computed with the following algorithm via SVD.

Forward TRR Algorithm: While $t_{k}<T$

$$
\begin{aligned}
& \text { Compute the SVD } \mathbf{G}_{k}^{*}=\mathbf{P}_{k} \mathbf{G}_{k} \mathbf{P}_{k}=\mathbf{U}_{k}^{t r} \operatorname{diag}\left(\lambda_{k j}^{\prime}\right) \mathbf{U}_{k} \\
& \mathbf{K}_{k}^{*} \leftarrow \mathbf{U}_{k}^{t r} \operatorname{diag}\left(\left(\lambda_{k j}^{\prime}\right)^{-1 / 2}\right) \mathbf{U}_{k} \mathbf{K}_{k} \mathbf{U}_{k}^{t r} \operatorname{diag}\left(\left(\lambda_{k j}^{\prime}\right)^{-1 / 2}\right) \mathbf{U}_{k} \\
& \text { Compute the } \operatorname{SVD} \mathbf{K}_{k}^{*}=\mathbf{V}_{k}^{t r} \operatorname{diag}\left(\lambda_{k j}^{\prime \prime}\right) \mathbf{V}_{k} \\
& \mathbf{f}_{k}(t) \leftarrow \mathbf{V}_{k}^{t r} \operatorname{diag}\left(\left(t_{k}+\lambda_{k j}^{\prime \prime}\right) /\left(t+\lambda_{k j}^{\prime \prime}\right)-1\right) \mathbf{V}_{k} \mathbf{U}_{k}^{t r} \operatorname{diag}\left(\left(\lambda_{k j}^{\prime}\right)^{-1 / 2}\right) \mathbf{U}_{k} \mathbf{g}\left(t_{k}\right) \\
& \boldsymbol{\beta}(t) \leftarrow \boldsymbol{\beta}\left(t_{k}\right)-\mathbf{U}_{k}^{t r} \operatorname{diag}\left(\left(\lambda_{k j}^{\prime}\right)^{-1 / 2}\right) \mathbf{U}_{k} \mathbf{f}_{k}(t), t \geq t_{k} \\
& \text { Compute } t_{k+1} \text { and } \mathbf{P}_{k+1} \text { from } \boldsymbol{\beta}(t), t>t_{k}
\end{aligned}
$$

\subsection{The Computational EfFiciency of the Forward GGD}

The computational cost of Forward GGD Algorithm I is essentially one or two SVD for each piece in $\left[t_{k}, t_{k+1}\right)$, so we focus on Forward GGD Algorithm II as an approximation of (1.2). The analysis of (6.8)-(6.11) and (5.1) as approximations of (5.4) is complicated, since the kernel $\mathbf{Q}_{k}(t)$ and knots $t_{k}$ are functions of the trajectory with possible discontinuities. Theorem 3 shows that (6.8)-(6.11) provide a much better approximation to (5.4) than (5.1) does within each interval of fixed $\mathbf{G}(t), \mathbf{P}(t)$ and $\mathbf{Q}(t)$. Let $\lambda_{\max }(\mathbf{H})$ be the largest eigenvalue of matrices $\mathbf{H}$.

Theorem 3. Suppose $\mathbf{G}(t)=\mathbf{G}_{0}, \mathbf{P}(t)=\mathbf{P}_{0}$ and $\mathbf{Q}(t)=\mathbf{Q}_{0}$ in $\left[t_{0}, T\right)$. Let $\mathbf{H}_{0}=$ $\mathbf{Q}(t) \mathbf{G}(t) \mathbf{P}(t)$. Let $\boldsymbol{\beta}(t)$ be the solution of (1.2) with a continuous measure $v(d t), \boldsymbol{\beta}\left(x_{j} ; \Delta\right)$ be as in (5.1) with $\Delta=v\left(\left(t_{0}, T\right]\right) / N$ and equal $v\left(x_{j+1}\right)-v\left(x_{j}\right)$, and $\widetilde{\boldsymbol{\beta}}(t ; N)$ be as in (6.7) with $k=0$, all with initialization $\boldsymbol{\beta}\left(t_{0}\right)\left(x_{0}=t_{0}\right)$. Then, for $\Delta \lambda_{\max }\left(\mathbf{H}_{0}\right) \leq 1$

$$
\max _{t_{0} \leq t \leq T}\|\widetilde{\boldsymbol{\beta}}(t ; N)-\boldsymbol{\beta}(t)\| \leq \frac{\Delta\left\{e \Delta \lambda_{\max }\left(\mathbf{H}_{0}\right)\right\}^{N}}{\{2 \pi(N+1)\}^{1 / 2}} \lambda_{\max }\left(\mathbf{Q}_{0}\right)\left\|\mathbf{g}\left(t_{0}\right)\right\|,
$$

and for small $\Delta \lambda_{\max }\left(\mathbf{H}_{0}\right)$,

$$
\boldsymbol{\beta}(t ; \Delta)-\boldsymbol{\beta}(t)=-(\Delta / 2) \mathbf{v}(t)+O(1) \Delta^{2} \lambda_{\max }\left(\mathbf{H}_{0}\right)\|\mathbf{v}(t)\|, \quad t=x_{0}, \ldots, x_{N},
$$

where $\mathbf{v}(t)=v\left(\left(t_{0}, t\right]\right) \mathbf{H}_{0}\left[\mathbf{H}_{0}\left\{\boldsymbol{\beta}(t)-\boldsymbol{\beta}\left(t_{0}\right)\right\}-\mathbf{Q}_{0} \mathbf{g}\left(t_{0}\right)\right]$.

Remark. Under the conditions of Theorem 3 , the computing cost for $\left\{\widetilde{\boldsymbol{\beta}}(t ; N), t_{0} \leq\right.$ $t \leq T\}$ and $\left\{\boldsymbol{\beta}(t ; \Delta), t=x_{0}, \ldots, x_{N}\right\}$ is nearly the same with $N \Delta=v\left(\left(t_{0}, T\right]\right)$, while (6.18) is of much smaller order than (6.19). In this sense, (6.8)-(6.11) provide a more accurate approximation to (1.2) than (5.1) does. The expansion in (6.19) provides the first order correction for the discrete algorithm (5.1) as an approximation of (1.2) for continuous $v$.

Proof. Let $\mathbf{H}_{0}^{*}=\mathbf{Q}_{0}^{1 / 2} \mathbf{G}_{0} \mathbf{Q}_{0}^{1 / 2}$ as in (6.2). Let $\mathbf{u}(t)=v\left(\left(t_{0}, t\right]\right) \mathbf{Q}_{0}^{1 / 2} \mathbf{g}\left(t_{0}\right)$. Since $\mathbf{H}_{0} \mathbf{Q}_{0}^{1 / 2}=\mathbf{Q}_{0}^{1 / 2} \mathbf{H}_{0}^{*}$, by (5.4) and (6.7)

$$
\boldsymbol{\beta}(t)-\widetilde{\boldsymbol{\beta}}(t ; N)=\left\{F^{*}\left(-v\left(\left(t_{0}, t\right]\right) \mathbf{H}_{0}\right)-F_{N}^{*}\left(-v\left(\left(t_{0}, t\right]\right) \mathbf{H}_{0}\right)\right\} v\left(\left(t_{0}, t\right]\right) \mathbf{Q}_{0} \mathbf{g}\left(t_{0}\right)
$$




$$
\begin{aligned}
& =\sum_{m=N+1}^{\infty}(m !)^{-1}\left\{-v\left(\left(t_{0}, t\right]\right) \mathbf{H}_{0}\right\}^{m-1} \mathbf{Q}_{0}^{1 / 2} \mathbf{u}(t) \\
& =\mathbf{Q}_{0}^{1 / 2} \sum_{m=N+1}^{\infty}(m !)^{-1}\left\{-v\left(\left(t_{0}, t\right]\right) \mathbf{H}_{0}^{*}\right\}^{m-1} \mathbf{u}(t) .
\end{aligned}
$$

Since $\left|\sum_{m=N+1}^{\infty} \lambda^{m-1} / m !\right| \leq \lambda^{m-1} / m$ ! for $-(N+1)<\lambda \leq 0$ and $\lambda_{\max }\left(v\left(\left(t_{0}, t\right]\right) \mathbf{H}_{0}^{*}\right)=$ $\lambda_{\max }\left(v\left(\left(t_{0}, t\right]\right) \mathbf{H}_{0}\right) \leq \lambda_{\max }\left(\mathbf{H}_{0}\right) N \Delta$, by the Stirling formula

$$
\begin{aligned}
\|\boldsymbol{\beta}(t)-\widetilde{\boldsymbol{\beta}}(t ; N)\| & \leq \lambda_{\max }\left(\mathbf{Q}_{0}^{1 / 2}\right) \lambda_{\max }\left(\left\{\mathbf{H}_{0}^{*} \nu\left(\left(t_{0}, t\right]\right\}^{N} /(N+1) !\right)\|\mathbf{u}(t)\|\right. \\
& \leq \lambda_{\max }^{2}\left(\mathbf{Q}_{0}^{1 / 2}\right) \lambda_{\max }^{N}\left(\mathbf{H}_{0}\right)(N \Delta)^{N+1}\left\|\mathbf{g}\left(t_{0}\right)\right\| /(N+1) ! \\
& \leq \Delta \lambda_{\max }\left(\mathbf{Q}_{0}\right)\left\{\lambda_{\max }\left(\mathbf{H}_{0}\right) \Delta\right\}^{N} \frac{N^{N+1}\left\|\mathbf{g}\left(t_{0}\right)\right\| e^{N+1}}{(N+1)^{N+1+1 / 2}(2 \pi)^{1 / 2}}
\end{aligned}
$$

This leads to $(6.18)$ since $(N /(N+1))^{N+1} \leq 1 / e$.

Now, let us prove (6.19). It follows from (5.1) and Condition I that

$$
\mathbf{P}_{0}\left\{\mathbf{g}\left(x_{j}\right)-\mathbf{g}\left(x_{j-1}\right)\right\}=-\mathbf{P}_{0} \mathbf{G}_{0}\left\{\boldsymbol{\beta}\left(x_{j} ; \Delta\right)-\boldsymbol{\beta}\left(x_{j-1} ; \Delta\right)\right\}=-\widetilde{\mathbf{H}}_{0} \mathbf{g}\left(x_{j-1}\right) \Delta
$$

with $\widetilde{\mathbf{H}}_{0}=\mathbf{P}_{0} \mathbf{G}_{0} \mathbf{Q}_{0}$, as in the proof of Theorem 2. It follows that

$$
\mathbf{P}_{0} \mathbf{g}\left(x_{j}\right)=\left(\mathbf{I}_{p}-\Delta \widetilde{\mathbf{H}}_{0}\right) \mathbf{P}_{0} \mathbf{g}\left(x_{j-1}\right)=\left(\mathbf{I}_{p}-\Delta \widetilde{\mathbf{H}}_{0}\right)^{j} \mathbf{P}_{0} \mathbf{g}\left(t_{0}\right)
$$

with $\mathbf{I}_{p}$ being the identity matrix in $\mathbb{R}^{p}$. Consequently,

$$
\begin{aligned}
\boldsymbol{\beta}\left(x_{j} ; \Delta\right) & =\boldsymbol{\beta}\left(x_{j-1} ; \Delta\right)+\mathbf{Q}_{0}\left(\mathbf{I}_{p}-\Delta \widetilde{\mathbf{H}}_{0}\right)^{j-1} \mathbf{P}_{0} \mathbf{g}\left(t_{0}\right) \Delta \\
& =\boldsymbol{\beta}\left(x_{j-1} ; \Delta\right)+\left(\mathbf{I}_{p}-\Delta \mathbf{H}_{0}\right)^{j-1} \mathbf{Q}_{0} \mathbf{g}\left(t_{0}\right) \Delta \\
& =\boldsymbol{\beta}\left(t_{0}\right)+F_{j}\left(-j \Delta \mathbf{H}_{0}\right)(j \Delta) \mathbf{Q}_{0} \mathbf{g}\left(t_{0}\right)
\end{aligned}
$$

with $F_{j}(\lambda)=\sum_{m=0}^{j-1}(1+\lambda / j)^{m} / j=\left\{(1+\lambda / j)^{j}-1\right\} / \lambda$. Let $r_{j}(\lambda)=\{1-(1+$ $\left.\lambda / j)^{j} e^{-\lambda}\right\} / \lambda$. Since $F^{*}(\lambda)=\left(e^{\lambda}-1\right) / \lambda$,

$$
F^{*}(\lambda)-F_{j}(\lambda)=\lambda^{-1}\left\{e^{\lambda}-(1+\lambda / j)^{j}\right\}=r_{j}(\lambda)\left\{\lambda F^{*}(\lambda)+1\right\} .
$$

Since $r_{j}(\lambda)=\lambda /(2 j)+O\left((\lambda / j)^{2}\right)$ and $j \Delta=v\left(\left(t_{0}, t\right]\right)$ at $t=x_{j}$, by Theorem 2

$$
\begin{aligned}
\boldsymbol{\beta}(t)-\boldsymbol{\beta}(t ; \Delta) & =\left\{F^{*}\left(-j \Delta \mathbf{H}_{0}\right)-F_{j}\left(-j \Delta \mathbf{H}_{0}\right)\right\}(j \Delta) \mathbf{Q}_{0} \mathbf{g}\left(t_{0}\right) \\
& =r_{j}\left(-j \Delta \mathbf{H}_{0}\right)\left\{-j \Delta \mathbf{H}_{0} F^{*}\left(-j \Delta \mathbf{H}_{0}\right)+1\right\}(j \Delta) \mathbf{Q}_{0} \mathbf{g}\left(t_{0}\right) \\
& =r_{j}\left(-j \Delta \mathbf{H}_{0}\right)(j \Delta)\left[-\mathbf{H}_{0}\left\{\boldsymbol{\beta}(t)-\boldsymbol{\beta}\left(t_{0}\right)\right\}+\mathbf{Q}_{0} \mathbf{g}\left(t_{0}\right)\right] \\
& =\left\{\Delta / 2+O(1) \Delta^{2} \mathbf{H}_{0}\right\} \mathbf{H}_{0} v\left(\left(t_{0}, t\right]\right)\left[\mathbf{H}_{0}\left\{\boldsymbol{\beta}(t)-\boldsymbol{\beta}\left(t_{0}\right)\right\}-\mathbf{Q}_{0} \mathbf{g}\left(t_{0}\right)\right]
\end{aligned}
$$

for small $\Delta \lambda_{\max }\left(\mathbf{H}_{0}\right)$. This implies (6.19) and completes the proof of Theorem 3.

\section{NUMERICAL EXPERIMENTS}

In this section, we report the results of three numerical experiments to demonstrate our implementation of the forward GGD and TRR algorithms described in Sections 6.1 and 6.2 . 

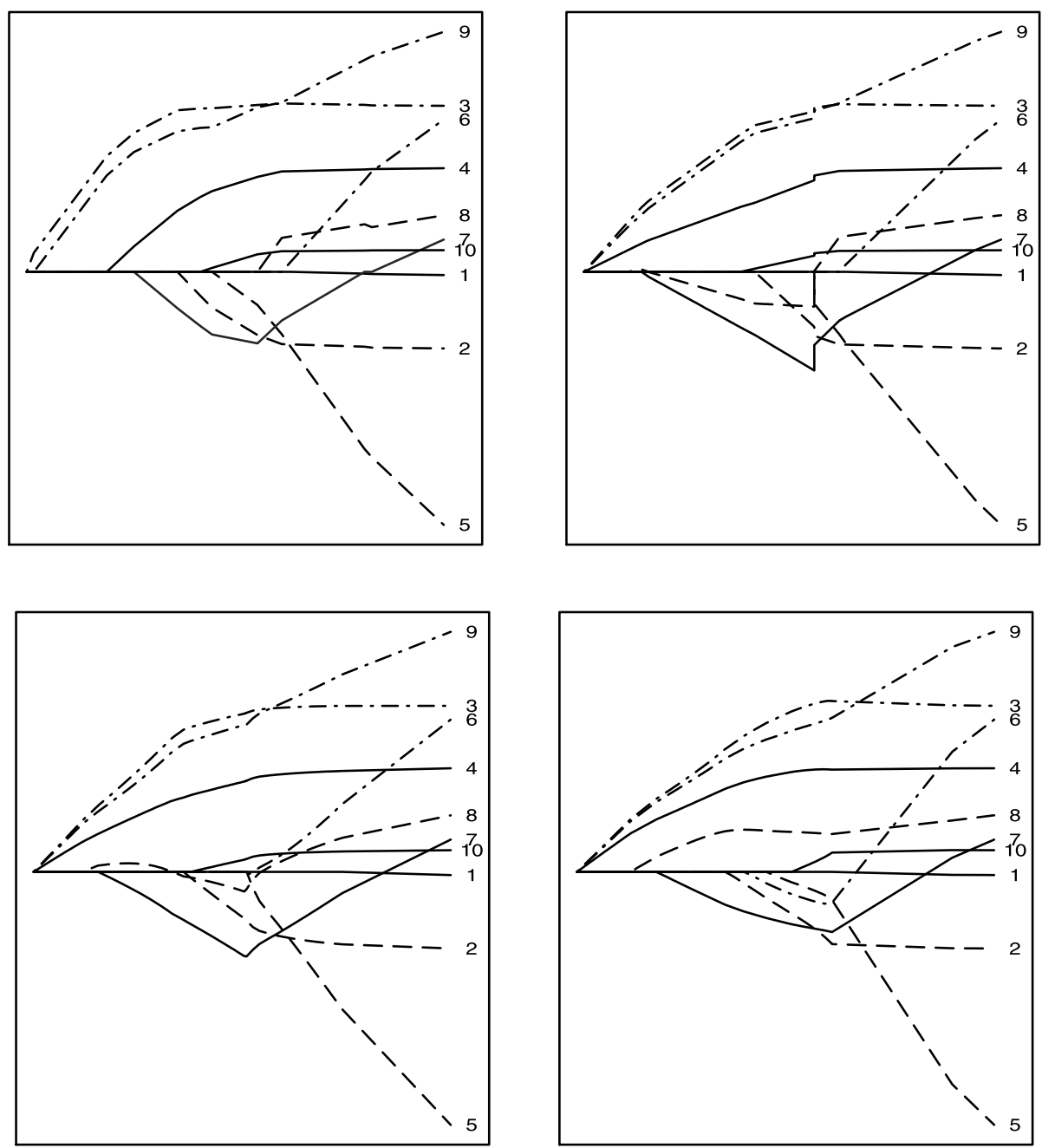

Figure 2. The trajectories of LASSO, PGS, NTGD, and NTRR (clockwise from top left) for the diabetes dataset with $p=10$ variables and sample size $n=422$. The $\beta_{j}(t)$ are plotted against the $\ell_{1}$ norm $\|\boldsymbol{\beta}(t)\|_{1}$.

In the first experiment, we run the PGS, NTGD, and nested TRR (NTRR), respectively given in (3.7), (2.10), and (4.15), on the diabetes dataset (see Efron et al. 2004), with the nested variable selector $\mathbf{P}_{3 / 4}^{*}(t)$ in (2.6). There are $p=10$ variables and $n=442$ observations. Forward GGD Algorithm I (6.1)-(6.6) is used to carry out the PGS and NTGD with $q(\lambda)=1 / \lambda$ and $q(\lambda)=1$, respectively in (4.12). Forward TRR Algorithm (6.12)-(6.17) is used to carry out the NTRR with the initialization $\boldsymbol{\beta}(1)=0$ at $t_{0}=1$. We plot the results against the $\ell_{1}$ norm $\|\boldsymbol{\beta}(t)\|_{1}$ in Figure 2 , along with LASSO.

In the second experiment, we add 1,000 additional variables $\mathbf{x}_{j}, j=11, \ldots, 1,010$ to the diabetes dataset, so that $p=1,010>n=442$. The additional vectors $\mathbf{x}_{j}$ are iid from the uniform distribution in $\left\{\mathbf{v} \in \mathbb{R}^{442}: \operatorname{mean}(\mathbf{v})=0, \operatorname{var}(\mathbf{v})=1\right\}$. We run the same four procedures as in the first experiment and exhibit their model selection properties in 
Table 1. Original variables added or dropped in listed steps. 1,000 simulated design variables are added to the diabetes dataset. LASSO misses more original variables than others.

\begin{tabular}{ccccccccccccc}
\hline \hline Actions & +1 & +2 & +3 & +4 & +5 & +6 & +7 & +8 & +9 & +10 & -5 & -6 \\
\hline Steps in PGS & 429 & 25 & 1 & 1 & 439 & 439 & 3 & 2 & 1 & 5 & \\
Steps in NTGD & 857 & 5 & 1 & 1 & 857 & 53 & 3 & 2 & 1 & 857 & \\
Steps in NTRR & 466 & 13 & 1 & 1 & 481 & 481 & 3 & 2 & 1 & 28 & \\
Steps in LASSO & & 27 & 1 & 3 & 345 & 493 & 4 & & 2 & & 365 & 506 \\
Steps in LASSO & & & & & 504 & 716 & & & & & 776 & \\
\hline
\end{tabular}

Table 1 . We see that all four trajectories select variables 3,4, 7, and 9 at the very beginning and variable 2 somewhat later, and they all dismiss variables 1, 5, and 6. While LASSO never selects variables 8 and 10, the three nested procedures all select variable 8 in Step 2 but disagree about variable 10 .

In the third experiment, we replace the response vector $\mathbf{y}$ by $1.645 \sum_{j=1}^{10} \mathbf{x}_{j}+\boldsymbol{\varepsilon}$, that is, $\beta_{j}=1.645 * I\{j \leq 10\}$, where $\varepsilon$ is a vector of iid $N(0,1)$ variables. We run the same four procedures as in the first two experiments on the dataset with this artificial response vector and the 1,010 covariate vectors $\mathbf{x}_{j}$ in the second experiment. We exhibit the model selection properties of the four trajectories in Table 2. We see that all four procedures select variables $1,2,3,4,6$, and 8 at the very beginning and dismiss variables 7 and 9 . While LASSO selects 5 at a late stage and never selects variable 10 , the three nested procedures all selected variables 5 and 10 early on.

\section{DISCUSSION}

In this article, we present the general gradient descent (GDD) (1.2) with continuous measure $v(d t)$ as a flexible and computationally tractable platform for multivariate estimation and model selection, with algorithms for the computation of the entire path of such trajectories. This includes as special cases the LASSO/LARS and proportional gradient shrinkage (PGS) in (3.7) as their extension, the nested threshold gradient descent (NTGD) in (2.10) motivated by the discrete TGD, the threshold ridge regression (TRR) in (4.15),

Table 2. Relevant variables added or dropped in listed steps. Simulated data with $p=1,010, n=422$, and $\beta_{j}=1.645 I\{j \leq 10\}$. The PGS, NTGD, and TRR outperform LASSO for model selection (for variables 5 and 10 ).

\begin{tabular}{cccccccccccc}
\hline \hline Actions & +1 & +2 & +3 & +4 & +5 & +6 & +7 & +8 & +9 & +10 & -6 \\
Steps in PGS & 3 & 2 & 1 & 1 & 1 & 1 & 445 & 1 & 445 & 6 & \\
Steps in NTGD & 3 & 2 & 1 & 1 & 1 & 1 & 860 & 1 & 618 & 4 & \\
Steps in NTRR & 3 & 2 & 1 & 1 & 1 & 1 & 512 & 1 & 337 & 6 & \\
Steps in LASSO & 6 & 5 & 2 & 3 & 86 & 4 & & 1 & & & 387 \\
\hline
\end{tabular}


and many more with any combinations of right-continuous variable selectors [e.g. (2.6), (3.6), (4.9), (4.10)], functional forms of the kernel $\mathbf{Q}(t)$ [e.g., (4.12), (4.13), (4.14)], and initializations. We prove the monotonicity and convergence properties of the proposed algorithms and their superior computational efficiency compared with their discretized versions with small steps. We demonstrate the applicability of our algorithms through numerical experiments with real and simulated datasets. However, investigation of probabilistic properties of these continuous GGDs is beyond the scope of this article.

For more general loss and penalty functions, the proposed algorithms can be used to generate piecewise analytic approximations of continuous GGD. For example, in a generalized linear model with negative log-likelihood

$$
L(\boldsymbol{\beta})=\sum_{i=1}^{n}\left\{\psi\left(\mathbf{x}_{i}^{t r} \boldsymbol{\beta}\right)-y_{i} \mathbf{x}_{i}^{t r} \boldsymbol{\beta}\right\},
$$

one may approximate the negative gradient with the discretization

$$
\mathbf{g}(t)=\sum_{i=1}^{n} \mathbf{x}_{i}\left(y_{i}-\psi^{\prime}\left(\mathbf{x}_{i}^{t r} \boldsymbol{\beta}\right)\right) \approx \sum_{i=1}^{n} \mathbf{x}_{i}\left(y_{i}-\mu_{m}\left(\mathbf{x}_{i}^{t r} \boldsymbol{\beta}\right)\right)
$$

with a first-order spline $\mu_{m}(t) \approx \psi^{\prime}(t)$. In this case, condition (2.9) holds and our algorithms apply. However, as in the case (6.7), the monotonicity property in Theorem 1 will be lost with such approximations. Discrete algorithms for the approximation of the trajectory (3.1) with the $\ell_{1}$ penalty in the generalized linear model (8.1) have been considered by Rosset (2004); Genkin, Lewis, and Madigan (2004); Zhao and Yu (2004); and Park and Hastie (2006), among others.

Friedman and Popescu (2004) suggested that one should adjust $\tau$ depending on the correlation among the predictors. In the continuous case, this means to use a new variable selector $\widetilde{\mathbf{P}}_{k}(t)$ at the time $t_{k}$. If $\mathbf{P}\left(t_{k}\right)=\widetilde{\mathbf{P}}_{k}\left(t_{k}\right)$, then we may just carry out our algorithms as is, with adjusted (6.6), (6.11) and (6.17) to find out the new $t_{k+1}$. Equivalently, one may simply define $\mathbf{P}(t)$ sequentially with $\mathbf{P}(t)=\widetilde{\mathbf{P}}_{k}(t)$ in $\left[t_{k}, t_{k+1}\right)$. If $\mathbf{P}\left(t_{k}\right) \neq \widetilde{\mathbf{P}}_{k}\left(t_{k}\right)$, one may redefine $\mathbf{P}\left(t_{k}\right)$ as $\widetilde{\mathbf{P}}_{k}\left(t_{k}\right)$, since $\mathbf{P}(t)$ is allowed to be discontinuous from the left. Another possibility is to allow the parameters $\{\tau, \widetilde{\tau}, \epsilon\}$ in (4.9) or (4.10) to depend continuously on the correlation of the active variables or even $\boldsymbol{\beta}(t)$ and $\mathbf{g}(t)$, as long as the resulting variable selector is still right-continuous.

[Received February 2006. Revised October 2006.]

\section{REFERENCES}

Breiman, L. (1996), "Heuristics of Instability and Stabilization in Model Selection,” The Annals of Statistics, 24, 2350-2383.

Efron, B., Hastie, T., Johnstone, I., and Tibshirani, R. (2003), "Least Angle Regression" (with discussion), The Annals of Statistics, 32, 407-499.

Freund, Y., and Schapire, R.E. (1996), Experiments with a New Boosting Algorithm. Machine Learning: Proceedings of the Thirteenth International Conference, San Francisco: Morgan Kauffmann, pp. 148-156. 
(1997), “A Decision-Theoretic Generalization of On-line Learning and an Application to Boosting," Journal of Computer System Science, 55, 119-139.

Friedman, J. (2001), "Greedy Function Approximation: A Gradient Boosting Machine," The Annals of Statistics, $29,1189-1232$.

Friedman, J. H., and Popescu, B. E. (2004), "Gradient Directed Regularization for Linear Regression and Classification," Stanford University, Department of Statistics, Technical Report.

Friedman, J., Hastie, T., and Tibshirani, R. (2000), "Additive Logistic Regression: A Statistical View of Boosting," (with discussion), The Annals of Statistics, 28, 337-307.

Genkin, A., Lewis, D., and Madigan, D. (2004), "Large-Scale Bayesian Logistic Regression for Text Categorization," available online at $h t t p: / / w w w . s t a t . r u t g e r s . e d u / \sim$ madigan/BBR/.

Hastie, T., Rosset, S., Tibshirani, R., and Zhu, J. (2004), "The Entire Regularization Path for the Support Vector Machine," Journal of Machine Learning Research, 5, 1391-1415.

Hoerl, A. E., and Kennard, R. (1970), "Ridge Regression: Biased Estimation for Nonorthogonal Problems,” Technometrics, 12, 55-67.

Osborne, M., Presnell, B., and Turlach, B. (2000a), "A New Approach to Variable Selection in Least Squares Problems," IMA Journal of Numerical Analysis, 20, 389-404.

(2000b), "On the Lasso and its Dual," Journal of Computational and Graphical Statistics, 9, 319-337.

Park, M.Y., and Hastie, T. (2006), "L1 Regularization Path Algorithm for Generalized Linear Models," http: //stat.stanford.edu/ hastie/pub.htm.

Rosset, S. (2004), "Tracking Curved Regularized Optimization Solution Paths," NIPS 2004.

Rosset, S., and Zhu, J. (2003), "Piecewise Linear Regularized Solution Paths," The Annals of Statistics, to appear.

Schapire, R. E. (1990), "The Strength of Weak Learnability," Machine Learning, 5, 197-227.

Tibshirani, R. (1996), "Regression Shrinkage and Selection via the Lasso," Journal of the Royal Statistical Society, Series B, 58, 267-288.

Vapnik, V. (1996), The Nature of Statistical Learning, New York: Springer-Verlag.

Wahba, G., Lin, Y., and Zhang, H. (2000), "Gacv for Support Vector Machines," in Advances in Large Margin Classifiers, eds. A.J. Smola, P.L. Bartlett, B. Schölkopf, and D. Schuurmans, Cambridge, MA: MIT Press, pp. 297-311.

Zhao, P., and Yu, B. (2004), “Boosted Lasso,” Tech Report, Statistics Department, U. C. Berkeley. 